\title{
Validation of the paraxial beam-tracing method in critical cases
}

\author{
Omar Maj, Grigory V. Pereverzev and Emanuele Poli \\ Max-Planck-Institut für Plasmaphysik, \\ EURATOM Association, Garching bei München, Germany
}

\begin{abstract}
Paraxial WKB (beam-tracing) solutions of a scalar wave equation are compared to the exact solutions for the two important cases of reflection from a plasma with linearly-increasing density and of oblique propagation in the presence of an absorbing half-plane. In these situations, the beam-tracing technique is close to its limits of validity, yet these conditions are often met in practical applications. More specifically, the first case is relevant to reflectometry diagnostics, whereas the second case models the absorption of electron-cyclotron beams obliquely launched onto the resonance layer, as envisaged, for instance, for the ITER upper launcher [M. A. Henderson et al., Nucl. Fusion 48, 054013 (2008)]. In both cases, the beam-tracing approach is found to reproduce well the exact behaviour of the wave for experimentally relevant parameter, confirming the robustness of WKB-based techniques close to or even beyond their range of applicability. For the latter case, moreover, the analysis presented here allows an evaluation of the reflection of part of the beam by the steep gradients of the absorption coefficient at the resonance layer, our results yielding an upper bound on the amount of reflected power.
\end{abstract}




\section{INTRODUCTION}

Asymptotic methods are employed to describe the propagation of waves in inhomogeneous media in many branches of physics and prove themselves extremely useful also in fusion research. When the typical inhomogeneity length $L$ characterizing the spatial variation of the medium is much larger than the wavelength $\lambda$, it is natural and convenient to study the asymptotics of the wave field in the short-wavelength limit $\lambda / L=\kappa^{-1} \rightarrow 0$; this leads to a simplified description of the wave propagation which is known as geometrical optics [1-3] or WKB approximation (after Wentzel-Kramers-Brillouin). To the lowest order in $1 / \kappa$, the wave beam is considered as a bundle of independent rays, traced according to Hamilton's equations in the position-wavevector phase space, the Hamiltonian function being the dispersion function of the considered wave. The phase (eikonal) of the wave field on each ray amounts to the action of such Hamiltonian system and the amplitude is also obtained on each ray by solving the appropriate transport equation. The solution of the wave equation is thus reduced to the integration of a set of ordinary differential equations. This represents a tremendous simplification of the initial problem. At this level, however, wave effects are not retained, so that ray tracing turns out to be unsatisfactory in all those applications for which diffraction effects are supposed to change in an essential way the behavior of the electromagnetic field. The simplest example is the propagation of a focussed beam, which in a simple ray tracing description exhibits at some point a caustic where its energy density diverges. Focussed or highly collimated beams are routinely used in fusion experiments for both heating and diagnostic purposes. For these reasons, more sophisticated asymptotic techniques that aim at retaining diffraction (the underlying ansatz being usually a replacement of the real eikonal with a complex eikonal [4-7]) have been developed [8-11]. These schemes are successfully implemented in numerical codes that calculate the propaga-

tion of Lower Hybrid (LH) [12, 13] and especially of Electron Cyclotron (EC) [14-16] waves in tokamaks.

The basic idea behind asymptotic techniques describing diffraction is that it is sufficient to retain wave effects in the direction transverse to the propagation of the beam, whereas such effects are neglected in the propagation direction. This introduces a new scale length, connected to the transverse beam width $W$, which is ordered $\lambda / W \approx W / L \approx \kappa^{-1 / 2}$. These considerations justify an approach to the problem in terms of a parabolic equation [17]. In 
this case, however, the most appealing property of geometrical optics, namely the reduction of the wave equation to a set of ordinary differential equations, is lost. A way to recover this feature is to make an explicit use of the ordering in semi-integer powers of $\kappa$, performing a paraxial expansion of the unknown parameters of the beam around a given curve, usually identified with the beam axis $[10,11]$. The coefficients of this expansion can be found integrating a set of ordinary differential equations. This approach is common in the theory of Gaussian beams [5, 6, 18-20], and it has been developed by Pereverzev [10, 11] for plasma physics applications where it is known as beam tracing (BT) or paraxial WKB (pWKB).

Although the conditions for the applicability of beam tracing are well satisfied in fusion plasmas in several scenarios, it is not difficult to imagine situations for which this approach fails. In this paper, we discuss two examples, particularly relevant under experimental conditions, for which the use of asymptotic solutions becomes critical [21], namely, the reflection from a cutoff layer and the oblique propagation in the presence of an absorbing halfplane. These two cases are intended to mimic, respectively, the typical set-up of reflectometry applications [22] and the case of oblique incidence onto a cyclotron resonance, as envisaged for instance for the ITER upper EC port [23, 24]. In order to shed light on the actual behavior of the wave and hence on the adequacy of asymptotic solutions, exact solutions of the Helmholtz equation have been obtained. As it will be explained later on in the paper, some aspects of the problem have been simplified to obtain equations which can be solved exactly with a reasonable computational effort. For instance, a planar 2D geometry has been considered in both cases. In the case of reflection from a cutoff, the density profile is assumed to vary linearly in space. The resonance layer has been approximated by a half-plane where a finite imaginary part is added to the vacuum refractive index. Nevertheless, the peculiar features of these problems, which make the use of asymptotic techniques questionable, are retained.

The capability of the beam tracing method of correctly describing the propagation and absorption of a wave beam in the two cases quoted above has been questioned recently $[25,26]$. Specifically, a criticism concerning the validity itself of the pWKB formulation of Refs. [10, 11] (which is also employed in the present paper) has been put forward in Ref. [25]. The claim is that the beam tracing equations derived in Refs. [10, 11] would fail to reproduce the beam profile in the case of propagation, e. g., in a "model isotropic medium with dielectric permittivity increasing linearly". It will be shown here (cf. Sec. III), 
on the basis of analytical and numerical results, that this criticism is completely unfounded, because it relies on an incorrect interpretation of the beam tracing equations.

As a matter of fact, it can be shown that the beam tracing method reproduces the solution of a the "parabolic wave equation" (which is, indeed, an equation of Schrödinger type) with a quadratic potential [11]. In this sense, solutions of the parabolic equation for a more generic potential, i. e., beyond the paraxial approximation, could lead to more reliable predictions. It has been suggested, for instance, that asymmetric beam absorption could indeed lead to broader absorption profiles under ITER conditions [26]. While the relevance of such "nonparaxial" effects cannot be excluded a priori, the results obtained with our simplified model (cf. Sec. IV) do not support this idea, showing that the beam-tracing absorption profiles reproduce very well the corresponding profiles obtained employing the exact wave field.

The scalar wave equation which is the starting point of the analysis presented in this paper is introduced in the next Section along with the corresponding energy continuity equation. The problem of reflection of a wave beam from a cutoff layer is analyzed in Sec. III, whereas the absorption of a wave beam by an absorbing layer for the case of oblique injection is discussed in Sec. IV. Concluding remarks can be found in Sec. V.

\section{BASIC EQUATIONS}

The purpose of this paper is the validation of the beam tracing description on the basis of exact solutions of the relevant partial differential equation satisfied by the wave field. To this aim, as stated in the Introduction, two experimentally relevant wave-propagation problems have been reduced to a simplified form, for which both exact and beam tracing solutions can be obtained.

Specifically, we consider electromagnetic waves with a fixed frequency $\omega$ that are described by the Helmholtz equation

$$
\Delta u+\frac{\omega^{2}}{c^{2}} n^{2}(x) u=0
$$

with a refractive index $n$ that is dependent on a single Cartesian coordinate $x$. For lossy media, $n^{2}(x)$ attains complex values, the (positive) imaginary part being the absorption coefficient. This equation can describe waves in isotropic slab-like media or ordinary waves in anisotropic plasmas with the external magnetic field orthogonal to the direction $\boldsymbol{e}_{x}$.

By applying the Helmholtz operator (1) to exponential wave functions $u(\boldsymbol{r})=e^{i \boldsymbol{k} \cdot \boldsymbol{r}}$ and 
multiplying by the complex conjugate $e^{-i \boldsymbol{k} \cdot \boldsymbol{r}}$ one obtains the local dispersion function,

$$
k_{0}^{2} H(x, \boldsymbol{k})=k^{2}-k_{0}^{2} n^{2}(x), \quad k_{0}=\frac{\omega}{c},
$$

which plays a major role in determining the behavior of high-frequency asymptotic solutions of equation (1), cf. Sec. III B. Since the squared refractive index $n^{2}$ depends on $x$ only, one can represent the solution by means of the Fourier transform in $(y, z)$ which gives

$$
\frac{\partial^{2} \hat{u}}{\partial x^{2}}+\left[k_{0}^{2} n^{2}(x)-k_{y}^{2}-k_{z}^{2}\right] \hat{u}=0
$$

$\hat{u}\left(x, k_{y}, k_{z}\right)$ being the Fourier-transformed field. We observe that the essential features of equations (1) and (3) are captured even if we restrict our analysis to beams comprising Fourier harmonics with $k_{z}=0$ only, so we can simplify the problem and consider the wave field as two-dimensional, i.e., $u(\boldsymbol{r})=u(x, y)$.

Let us recall that a solution of the Helmholtz equation (1) satisfies the energy continuity equation

$$
\operatorname{div} \boldsymbol{S}=-k_{0} \gamma|u|^{2}, \quad \gamma=\operatorname{Im}\left\{n^{2}\right\}
$$

where

$$
\boldsymbol{S}=\frac{1}{2 i k_{0}}\left(u^{*} \nabla u-u \nabla u^{*}\right)
$$

The energy conservation law (4) follows directly from the Helmholtz equation (1): one can multiply by the complex conjugate of the field $u^{*}$, subtract from the result its complex conjugate and exploit the identity $u^{*} \Delta u-u \Delta u^{*}=\operatorname{div}\left(u^{*} \nabla u-u \nabla u^{*}\right)$. Alternatively, one can note that the flux vector (5) is (proportional to) the Poynting vector with $\boldsymbol{E}=u \boldsymbol{e}_{z}$ and $i k_{0} \boldsymbol{B}=\operatorname{curl} \boldsymbol{E}$.

In Sections III and IV two specific profiles of the refractive index $n^{2}$ will be addressed. Specifically, in Sec. III, $n^{2}$ is real valued and decreases linearly from positive to negative values, therefore no absorption takes place and a cutoff layer is present where $n^{2}=0$. In Sec. IV, we take a piecewise constant profile which models a strong wave absorption in a half-space. In both cases we shall work out the exact solution and describe briefly the construction of the corresponding beam tracing solution. In both cases, since the beam tracing technique is at the limit (or beyond) its formal applicability, the analysis performed in this paper allows us to determine the reliability of the asymptotic pWKB solutions in practically significant cases. 


\section{REFLECTION BY A LINEAR LAYER}

Beam tracing techniques are used in reflectometry applications [22, 27] since they allow a very fast determination of the beam path, which would otherwise require a much more computationally demanding full-wave approach. In a typical reflectometry scenario, a microwave beam is launched into the plasma with the frequency tuned so that there exists a cutoff from which the beam is reflected; for such beams, however, we are very close to the limits for the applicability of the beam tracing method, especially near the point where the reflection takes place.

The paradigm for the propagation near a cutoff is the linear-layer problem, that is the Helmholtz equation (1) with the real-valued linear refractive index

$$
n^{2}(x)=1-x / L, \quad k^{2}(x)=k_{0}^{2}(1-x / L),
$$

where $L$ is the scale length of the medium and represents the distance between free space $n^{2}=1$ and the cutoff $n^{2}=0$.

\section{A. Exact solution}

In view of the specific form of the refractive index (6), it is convenient to introduce rescaled coordinates $x^{\prime}=x / L, y^{\prime}=y / L$. Correspondingly, we introduce the normalized frequency $\kappa=k_{0} L=\omega L / c \gg 1$, which is the large parameter of the standard WKB method. The problem is well studied and described in detail in Ginzburg's book [28]. Following this reference, we first present the exact solution of (1) with (6) by the Fourier integral

$$
u^{E x}\left(x^{\prime}, y^{\prime}\right)=\frac{\kappa}{2 \pi} \int_{-\infty}^{+\infty} e^{i \kappa N_{y} y^{\prime}} \hat{u}\left(x^{\prime}, N_{y}\right) \mathrm{d} N_{y},
$$

the integration being over the $y$-component $N_{y}=c k_{y} / \omega$ of the refractive index vector. On substituting (7) into (1) one finds the spectral function $\hat{u}\left(x^{\prime}, N_{y}\right)$ as the solution of the Airy equation in the form

$$
\hat{u}\left(x^{\prime}, N_{y}\right)=f\left(N_{y}\right) \operatorname{Ai}\left(-\kappa^{2 / 3}\left(1-x^{\prime}-N_{y}^{2}\right)\right) .
$$

where $f\left(N_{y}\right)$ is an arbitrary function, the Airy integral is given by

$$
\operatorname{Ai}(-\xi)=\frac{1}{\pi} \int_{0}^{\infty} \cos \left(\frac{\eta^{3}}{3}-\xi \eta\right) \mathrm{d} \eta
$$


and its argument $\xi=\kappa^{2 / 3} N_{x}^{2}$ is related to the $x$-component of the refractive index $N_{x}^{2}=$ $1-x^{\prime}-N_{y}^{2}$. Equation (8) gives a bounded particular solution of the linear layer problem defined within an arbitrary factor $f\left(N_{y}\right)$. The factor has to be chosen in order to pick up the solution corresponding to the considered physical problem.

In order to understand the physical meaning of the solution (8) we consider the high frequency limit $\kappa=\omega L / c \gg 1$. Lowest order asymptotic terms of the Airy function $\operatorname{Ai}(-\xi)$ at $|\xi| \gg 1$ give [28, 29]

$$
\operatorname{Ai}(-\xi) \approx \frac{\xi^{-1 / 4}}{2 \sqrt{\pi}} \begin{cases}2 \cos \left(\frac{2}{3} \xi^{3 / 2}-\frac{\pi}{4}\right), & \text { for } \xi>0 \\ \exp \left(-\frac{2}{3}|\xi|^{3 / 2}\right), & \text { for } \xi<0\end{cases}
$$

It is now seen that the expressions in brackets on the right hand side of (9) can be thought

of as $\xi^{3 / 2} \propto \int \sqrt{1-x^{\prime}-N_{y}^{2}} \mathrm{~d} x^{\prime}= \pm \int N_{x}\left(x^{\prime}\right) \mathrm{d} x^{\prime}$ and thus coincide with the eikonal of the geometric-optics solution.

At $x^{\prime} \leq 1-N_{y}^{2}$, when $N_{x}$ is real valued, the solution is oscillatory. Two real valued roots $N_{x}= \pm \sqrt{1-x^{\prime}-N_{y}^{2}}$ correspond to the waves $e^{ \pm i \kappa \frac{2}{3}\left(1-x^{\prime}-N_{y}^{2}\right)^{3 / 2}}$ travelling in opposite directions. Moreover, if the pre-factor $f\left(N_{y}\right)$ in (8) is not even, these two waves are separated in space. Otherwise, Eq. (8) describes a standing wave. On the other hand, when $N_{x}$ is complex valued, i.e., $x^{\prime}>1-N_{y}^{2}$, the wave is evanescent. The expression (8) represents the spectral distribution of the exact solution in each plane $x=$ Const. Any bounded solution of Eqs. (1), (6) can be represented in this form by an appropriate choice of the arbitrary function $f\left(N_{y}\right)$. In Sec. IIIC, this freedom will be used in order to pick up the specific solution with the same asymptotic behaviour as the BT solution. It is also worth to note here that the phase shift between the incident and the reflected waves is $\pi / 2$. This phase shift is not described by the geometrical optics (in the form used in applications, i.e., without the Maslov index) but will be found in the BT solution as discussed in the next Section.

\section{B. Beam tracing solution}

The beam tracing description of wave beams $[10,11]$ resembles the paraxial approach to complex geometric optics, which has been applied to the linear layer problem by Kravtsov and Berczynski [30].

The very basis of such paraxial techniques is the strong localization of the wave field 


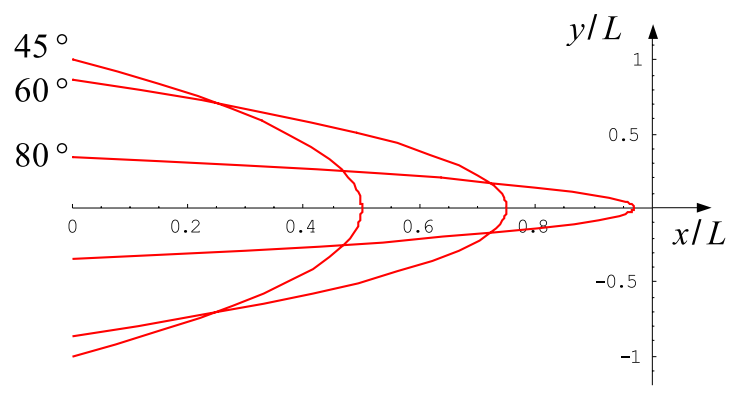

FIG. 1: The reference ray obtained from the solution of Hamilton's equations (10) for $\theta=45^{\circ}$, $60^{\circ}$, and $80^{\circ}$.

around the trajectory of the beam which can be identified with the geometrical optics ray issuing from the point where the maximum field intensity is launched. The ray trajectory is called reference ray and it is computed by means of Hamilton's equations,

$$
\frac{\mathrm{d} q^{\alpha}}{\mathrm{d} \tau}=\frac{\partial H}{\partial N_{\alpha}}, \quad \frac{\mathrm{d} N_{\alpha}}{\mathrm{d} \tau}=-\frac{\partial H}{\partial q^{\alpha}}, \quad \alpha=1,2,
$$

where $\boldsymbol{q}=\left(q^{\alpha}\right)=\left(x^{\prime}, y^{\prime}\right)$ is the normalized position, $\boldsymbol{N}=\left(N_{\alpha}\right)=\left(N_{x}, N_{y}\right)$ is the refractive index vector which plays the role of conjugate momentum, and $\tau$ is the parameter along the ray; moreover, $H(\boldsymbol{q}, \boldsymbol{N})=N^{2}-1+x^{\prime}$ is the Hamiltonian (2) normalized to $k_{0}^{2}$. The initial conditions for (10) are conveniently written in the form $\boldsymbol{q}(0)=\left(x_{0}^{\prime}, y_{0}^{\prime}\right)=(0, \sin 2 \theta)$, $\boldsymbol{N}(0)=\left(N_{x 0}, N_{y 0}\right)=(\sin \theta,-\cos \theta)$, with $0<\theta<\pi / 2$ being the beam injection angle, i.e., the counter-clockwise angle between the $x$-axis and the "velocity" vector $\mathrm{d} \boldsymbol{q} / \mathrm{d} \tau=2 \boldsymbol{N}$ in the initial position $\boldsymbol{q}(0)$; the dispersion equation $H=0$ is satisfied by such initial conditions and, thus, it is satisfied on the whole solution which reads

$$
\begin{array}{ll}
x^{\prime}(\tau)=x_{0}^{\prime}+2 N_{x 0} \tau-\tau^{2}, & N_{x}(\tau)=N_{x 0}-\tau, \\
y^{\prime}(\tau)=y_{0}^{\prime}+2 N_{y 0} \tau, & N_{y}(\tau)=N_{y 0},
\end{array}
$$

where the curve $\boldsymbol{q}(\tau)=\left(x^{\prime}(\tau), y^{\prime}(\tau)\right)$ is the reference ray together with the carried refractive index $\boldsymbol{N}(\tau)=\left(N_{x}(\tau), N_{y}(\tau)\right)$. The reference ray, in particular, amounts to a parabola symmetric with respect to the $x$-axis and it is narrower for values of $\theta$ closer to $\pi / 2$; in the limit case $\theta=\pi / 2$ the reference ray degenerates to two superposed segments of a straight line. The "classical" turning point is defined by the condition $\tau=N_{x 0}=\sin \theta$. Fig. 1 shows the reference ray for three values of $\theta$.

The main point in the construction of the beam tracing solution is the evolution along the reference ray of the symmetric complex matrix $\Psi_{\alpha \beta}=N_{\alpha \beta}+i \phi_{\alpha \beta}, \alpha, \beta=1,2$, which 
satisfies the complex matrix Riccati equation [11, Eq.(97)],

$$
\frac{\mathrm{d} \Psi_{\alpha \beta}}{\mathrm{d} \tau}+\frac{\partial^{2} H}{\partial q^{\alpha} \partial q^{\beta}}+\frac{\partial^{2} H}{\partial q^{\alpha} \partial N_{\gamma}} \Psi_{\gamma \beta}+\Psi_{\alpha \gamma} \frac{\partial^{2} H}{\partial N_{\gamma} \partial q^{\beta}}+\frac{\partial^{2} H}{\partial N_{\mu} \partial N_{\nu}} \Psi_{\nu \alpha} \Psi_{\mu \beta}=0,
$$

along with the constraints

$$
\frac{\partial H}{\partial q^{\alpha}}+\Psi_{\alpha \gamma} \frac{\partial H}{\partial N_{\gamma}}=0
$$

where all the derivatives of the Hamiltonian function $H$ should be evaluated on the reference ray and the sum over repeated indices is implied. With the solutions for the reference ray and the matrix $\Psi_{\alpha \beta}$ at our disposal, the beam tracing solution for a Gaussian beam is readily obtained in the form $[10,11]$

$$
u^{B T}(\boldsymbol{r})=u_{0} e^{-\int_{0}^{\tau} \operatorname{Tr} \Psi_{\alpha \beta}\left(\tau^{\prime}\right) \mathrm{d} \tau^{\prime}} e^{i \kappa}\left[\int_{0}^{\tau} N_{\alpha}\left(\tau^{\prime}\right) \mathrm{d} q^{\alpha}\left(\tau^{\prime}\right)+N_{\alpha}(\tau) \cdot\left(q^{\alpha}-q^{\alpha}(\tau)\right)+\frac{1}{2} \Psi_{\alpha \beta}(\tau)\left(q^{\alpha}-q^{\alpha}(\tau)\right)\left(q^{\beta}-q^{\beta}(\tau)\right)\right],
$$

where Tr denotes the trace of a matrix, and $\tau=\tau(\boldsymbol{q})$ is the value of the parameter along the reference ray corresponding to the point $\boldsymbol{r}=L \boldsymbol{q}$ where the field is evaluated; more specifically, the function $\tau(\boldsymbol{q})$ is obtained by inverting the expression $\boldsymbol{q}=q(\tau)+\boldsymbol{e}(\tau) \eta$, where $(\tau, \eta)$ are viewed as local coordinates around the reference ray, $\boldsymbol{e}(\tau)$ being a unit vector skewed with respect to the tangent to the reference ray. There is some degree of freedom in the choice of the unit vector $\boldsymbol{e}(\tau)$, and thus in the function $\tau(\boldsymbol{q})$, as it is discussed in details in the appendix along with a convenient choice for the linear layer problem under consideration.

The physical content of (14) becomes evident upon separating the real and imaginary parts of $\Psi_{\alpha \beta}=N_{\alpha \beta}+i \phi_{\alpha \beta}$. Then, one can see that the exponential $e^{-\kappa \phi}$, with $\phi=\frac{1}{2} \phi_{\alpha \beta}\left(q^{\alpha}-\right.$ $\left.q^{\alpha}(\tau)\right)\left(q^{\beta}-q^{\beta}(\tau)\right)$, accounts for the Gaussian envelope of the beam, therefore, the matrix $\phi_{\alpha \beta}$ encodes the information on the beam width; explicit formulas are given in appendix. On the other hand, the oscillatory exponential $e^{i \kappa S}$, with phase $S=\int_{0}^{\tau} N_{\alpha}\left(\tau^{\prime}\right) \mathrm{d} q^{\alpha}\left(\tau^{\prime}\right)+N_{\alpha}(\tau)$. $\left(q^{\alpha}-q^{\alpha}(\tau)\right)+\frac{1}{2} N_{\alpha \beta}(\tau)\left(q^{\alpha}-q^{\alpha}(\tau)\right)\left(q^{\beta}-q^{\beta}(\tau)\right)$, describes the fast oscillations of the wave field and its phase-front geometry in terms of the sum of the geometric optics phase on the reference ray (the action integral for the corresponding Hamiltonian system), plus the effect of the angle between the phase fronts and the direction $\boldsymbol{e}(\tau)$ (the linear term) and the effects of the curvature of the phase fronts (the quadratic form associated to $N_{\alpha \beta}$ ); the quadratic form $N_{\alpha \beta}$, in particular, encodes the information on the phase-front radius of curvature as discussed in appendix. One can also see that the exponential associated to the trace $\operatorname{Tr} \Psi$ 
splits into a real amplitude $C$, which accounts for the amplitude ramp up/down due to the focussing/defocussing of the beam, times a phase shift factor which is just the Gouy shift also present in the exact solution, cf. comments at the end of Sec. III A.

Let us conclude this section with two remarks on the validity of the beam tracing solution (14) for the specific case of the linear layer problem under consideration.

It is known that the geometric-optics solution of the problem of reflection from a cutoff layer exhibits an unphysical singularity near the turning point. In principle, the BT solution (14) does not present this problem. However, some restrictions are also set to the applicability of the pWKB method, as emerges from the following considerations. The wave object (14) is an asymptotic solution of the Helmholtz equation (1) in the high-frequency limit $\kappa \rightarrow+\infty$, and, since $w=O(1 / \sqrt{\kappa}), u^{B T}$ collapses on the reference ray in that limit. For finite (but large) value of $\kappa \gg 1$, i.e., in the applications, $u^{B T}$ solves approximately the Helmholtz equation (1) in a strip of (finite) width $w$ about the reference ray, provided that the local coordinates $(\tau, \eta)$ are well-defined in that strip; heuristically, this means that the ratio $w / R_{\text {ray }}$ between the beam width and the radius of curvature of the reference ray should be small enough, specifically, $w / R_{\text {ray }} \approx 1 / \sqrt{\kappa} \ll 1$. Even in smoothly inhomogeneous media, however, it can happen that $R_{\text {ray }}$ is comparable or even shorter than $w$, and for such beams the validity of the beam tracing solution (14) becomes questionable. In the case under consideration, the more the injection angle $\theta$ is close to $\pi / 2$, the narrower is the parabola of the reference ray, making the geometry more and more critical; in the limit case $\theta=\pi / 2$ the beam tracing construction is no longer applicable at all.

At last, let us note that, for the problem under consideration, the only non-trivial secondorder derivatives of the Hamiltonian are $\partial^{2} H / \partial N_{\alpha} \partial N_{\beta}=2 \delta^{\alpha \beta}$, hence, the Riccati equation (12) is the same as the one relevant to the free-space diffraction problem $(n(x) \equiv 1)$, all the properties of the medium being accounted for by the constraint (13). On the basis of the formal analogy of (12) with free space propagation, Balakin et al. [25] argued that linear profiles of the refractive index cannot be properly described in the beam tracing framework, cf. also the Introduction; such an argument, however, is flawed as it does not account for the constraint (13) which changes the initial conditions in an essential way and couples the components of the matrix $\Psi_{\alpha \beta}$. 


\section{Comparison of BT and exact solutions}

The aim of this Section is the validation of the beam tracing solution (14) for different values of the input parameters.

With this aim, the following spectrum of the launched beam is considered,

$$
\hat{U}\left(N_{y}\right)=u_{0} \sqrt{\frac{2 \pi}{\kappa \mu}} e^{-\frac{\kappa}{2 \mu}\left(N_{y}-N_{y 0}\right)^{2}} e^{-i \kappa N_{y} y_{0}^{\prime}},
$$

where $\mu=2 / \alpha^{2}-i \beta$ is a complex parameter. The spectrum (15) is just the Fourier transform of the section $x^{\prime}=x_{0}^{\prime}=0$ of the launched beam which is taken in the form $u_{0} e^{-\kappa\left(y^{\prime}-y_{0}^{\prime}\right)^{2} / \alpha^{2}} e^{i \kappa\left[N_{y 0}\left(y^{\prime}-y_{0}^{\prime}\right)+\frac{1}{2} \beta\left(y^{\prime}-y_{0}^{\prime}\right)^{2}\right]}, \alpha / \sqrt{\kappa}$ being, thus, the normalized width of the beam section at $x=0$ and $\beta$ the projected curvature of the phase front; it is worth noting that, because of the diffraction of the beam, $\alpha$ and $\beta$ are related to the normal width $w_{0}=w(0)$ (normalized to $L$ ) and phase-front curvature $1 / R_{0}=1 / R(0)$ (normalized to $L$ ) in a nontrivial way, namely,

$$
\alpha=\frac{\sqrt{\kappa}}{\sin \theta} w_{0}, \quad \beta=\sin ^{2} \theta\left(\frac{1}{R_{0}}+\frac{\cos ^{2} \theta}{\sin \theta}\right) .
$$

The sketch of the derivation of equation (16) is given in appendix along with the precise definitions of the beam width $w(\tau)$ and phase-front curvature radius $R(\tau)$.

The particular exact solution corresponding to (15) is obtained by selecting the multiplier $f\left(N_{y}\right)$ in (8) in such a way that the forward-propagating component, identified through the asymptotic expansion (9) and evaluated at $x=0$, equals the launched spectrum (15); that yields

$$
f\left(N_{y}\right)=2 \sqrt{\pi} \kappa^{1 / 6}\left(1-N_{y}^{2}\right)^{1 / 4} e^{i \frac{2}{3}\left(1-N_{y}^{2}\right)^{3 / 2}-i \pi / 4} \hat{U}\left(N_{y}\right)
$$

for propagating harmonics $\left(N_{y}^{2} \leq 1\right)$, and $f\left(N_{y}\right)=0$ for evanescent harmonics $\left(N_{y}^{2}>1\right)$.

As for the beam tracing solution, in order to match the launched beam profile it is convenient to pick up adapted local coordinates; specifically, we set, cf., comments after equation (14) and the appendix, $\boldsymbol{e}=\left(e_{x}, e_{y}\right)$ with $e_{x} \propto-\cos \theta\left(\sin \theta \tau-\tau^{2} / 2\right)$ and $e_{y} \propto$ $\tau-\sin \theta$, properly normalized; according to such a definition, both the axes $\{\tau=0\}$ and $\{\tau=2 \sin \theta\}$ coincide with the $y$-axis, with the latter having opposite orientation, while $\{\tau=\sin \theta\}$ coincides with the $x$-axis (opposite orientation); it is implied that the coordinates $(\tau, \eta)$ has a local validity near the reference ray; nonetheless, away from the reference ray the wave field is exponentially small. 
With this choice, the section $\tau=0$ of the beam tracing solution (14) matches the launched field provided that $\Psi_{22}(0)=i \mu \neq 0$; the constraints (13) then give the initial conditions for the remaining components of $\Psi_{\alpha \beta}$. As mentioned above, in our special case the Riccati equation (12) takes the simple form

$$
\frac{\mathrm{d} \Psi_{\alpha \beta}}{\mathrm{d} \tau}+2 \Psi_{\alpha \nu} \Psi_{\mu \beta} \delta^{\nu \mu}=0
$$

and can easily be solved analytically. On account of (13), one finds $\operatorname{det}\left\|\Psi_{\alpha \beta}\right\|=$ $-i \mu /\left(2 N_{x}\right) \neq 0$ and concludes that, in the vicinity of the starting point $\tau=0$, the matrix $\Psi_{\alpha \beta}(0)$ has an inverse $\Phi^{\alpha \gamma} \Psi_{\gamma \beta}=\delta_{\beta}^{\alpha}$. Multiplying Eq. (18) by $\Phi^{\alpha \rho} \Phi^{\beta \gamma}$ yields $\mathrm{d} \Phi^{\rho \gamma} / \mathrm{d} \tau=2 \delta^{\rho \gamma}$ that gives the general solution $\Phi^{\alpha \beta}(\tau)=\Phi^{\alpha \beta}(0)+2 \tau \delta^{\alpha \beta}$ with only one of the set of complex valued constants $\Phi^{\alpha \beta}(0)$ being independent. Then, matrix $\Psi_{\alpha \beta}(\tau)$ is readily computed by inverting the $2 \times 2$ matrix $\Phi^{\alpha \beta}(\tau)$. The element $\Psi_{11}(\tau)$, in particular, amounts to

$$
\Psi_{11}(\tau)=\frac{\tau-N_{y 0}^{2} / N_{x 0}-(i / 2) \mu^{-1}}{2 \tau^{2}-2 \tau / N_{x 0}-i \tau / \mu+i N_{x 0} / \mu} .
$$

When evaluated at the classical turning point $\tau=N_{x 0}, \Psi_{11}$ is directly related to the beam width $w\left(N_{x 0}\right)$ defined in the appendix: the only non-zero element of the matrix $\Psi_{\alpha \beta}\left(N_{x 0}\right)$ is exactly $\Psi_{11}\left(N_{x 0}\right)$ which, therefore, equals $2 /\left(\kappa w^{2}\left(N_{x 0}\right)\right)$. The beam width at the turning is then written analytically as

$$
w_{\mathrm{tp}}(\theta)=w\left(N_{x 0}\right)=\frac{4}{\alpha}\left[\frac{1+\alpha^{4} \beta^{2} / 4}{\kappa}\right]^{\frac{1}{2}} \cos \theta .
$$

We can see that $w_{t p}$ decreases as the injection angle approaches the critical value $\theta=90^{\circ}$, in correspondence of which it equals zero, and, neglecting $\alpha^{4} \beta^{2} / 4 \ll 1, w_{\text {tp }} \sim 1 / \alpha$, i.e., according to the beam tracing solution, an initially wider beam is narrower at the classical turning point.

We note that both the exact solution (7) and the beam tracing solution (14) are complexvalued; for the linear layer model, however, the refractive index $n^{2}(x)$ is real, hence, $\operatorname{Re}\left(u^{E x}\right)$ and $\operatorname{Re}\left(u^{B T}\right)$ are real-valued exact and beam tracing solutions, respectively. In Fig. 2, these two quantities are represented for typical values of the parameters used in Doppler reflectometry on the ASDEX Upgrade tokamak [22]. In Fig. 2a, we can see a non trivial diffractive pattern near the turning point of the beam, due to the superposition of the tails of the wave field. The contour plot in Fig. 2 shows a rather good matching of the exact and beam tracing solutions, in both the phase and the amplitude, yet the diffractive pattern is 

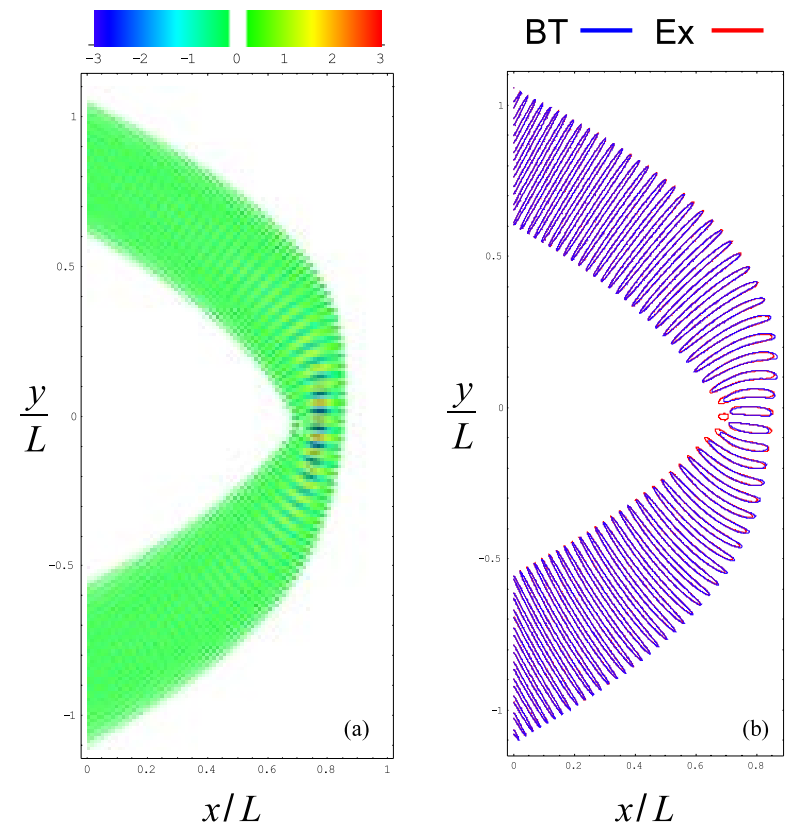

FIG. 2: (Color online) The normalized exact solution $\operatorname{Re}\left(u^{E x} / u_{0}\right)$ represented by a density plot (a), and the contours $\operatorname{Re}\left(u / u_{0}\right)=0.2$ for both the exact and the beam tracing solution (b); the color code in (a) is cut out for $\left|\operatorname{Re}\left(u / u_{0}\right)\right|<0.2$ so that a direct comparison with (b) is possible. The parameters are typical for Doppler reflectometry at ASDEX Upgrade: $\kappa=236.65(\omega / 2 \pi=64$ GHz), $\theta=62^{\circ}, \alpha=0.18 \sqrt{\kappa}(w=0.18 L$ being the physical beam width), and $\beta=0.0346$.

excluded in the beam tracing solution. This is due to the local character of the beam tracing construction which is sensitive to the field structure near the reference ray only, and, thus, it cannot account for the superposition of the wave-field tails; on the other hand such effects are exponentially small when the curvature radius of the reference ray is large enough.

Before going through a quantitative analysis of such effects, further insights are given in Fig. 3, where the amplitude contours of the beam tracing solution and their (absolute) error are shown. The external (low-amplitude) contour in Fig. 3a exhibits a bottleneck-like profile near the turning point which corresponds to the absence of the diffractive pattern; on the other hand, the inner (high-amplitude) contour shows qualitatively the position and the size of the caustic where the wave-field intensity is stronger. For the purposes of reflectometry the caustic is the region where a significant interaction of the wave beam with the underlying plasma density fluctuations takes place [31], therefore it is particularly important to estimate its position and size that are related to the spatial resolution of the reflectometry diagnostic. 

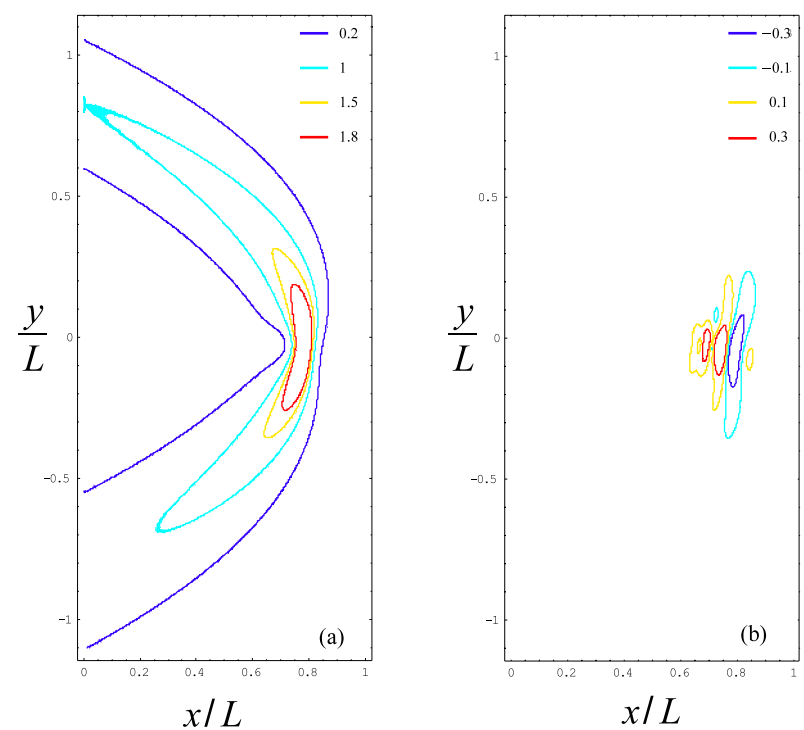

FIG. 3: (Color online) Contours of the beam tracing amplitude $\left|u^{B T} / u_{0}\right|,(\mathrm{a})$, and their absolute error $\left|u^{E x} / u_{0}\right|-\left|u^{B T} / u_{0}\right|$, (b), for the same parameters used in Fig. 2.

The (absolute) error, shown in Fig. 3b, is localized at the turning point mainly due to the lack of diffractive pattern in the beam tracing solution.

For a quantitative description of the position and size of the caustic, let us consider a sample of beams defined as follows. The frequency is fixed, $\kappa=200$, for all beams in the sample and so is the focussing parameter, $\beta=-0.05$; three values of the initial width are considered, namely, $\alpha / \sqrt{\kappa}=0.1,0.15,0.2$ and the injection angle sweeps from $\theta=55^{\circ}$ to $\theta=80^{\circ}$ with step-size $\Delta \theta=5^{\circ}$. These values range around relevant reflectrometry configurations. For each beam, the exact solution and the beam tracing solution have been computed together with a "generalized" version of the beam tracing solution which allows us to describe the superposition of the incident and reflected beams, and thus the diffractive pattern shown in Fig. 2. The idea of such a generalization is the following (we refer to the appendix for more technical details). As a consequence of the geometry of the reference ray, by inverting the relation $q^{\alpha}(\tau, \eta)=q^{\alpha}(\tau)+e^{\alpha}(\tau) \eta$ in correspondence of each point $\boldsymbol{q}=\left(q^{\alpha}\right)$ in the plane, one finds a cubic equation for $\tau(\boldsymbol{q})$ which can have either a single real root $\tau(\boldsymbol{q})$ or three real roots $\tau_{j}(\boldsymbol{q}), j=1,2,3$; in the regions of the plane where three roots are found, one can identify the two of them that correspond to the incident and reflected branches of the beam, say, $\tau_{1}(\boldsymbol{q})$ and $\tau_{2}(\boldsymbol{q})$, then, define the generalized beam tracing solution as 

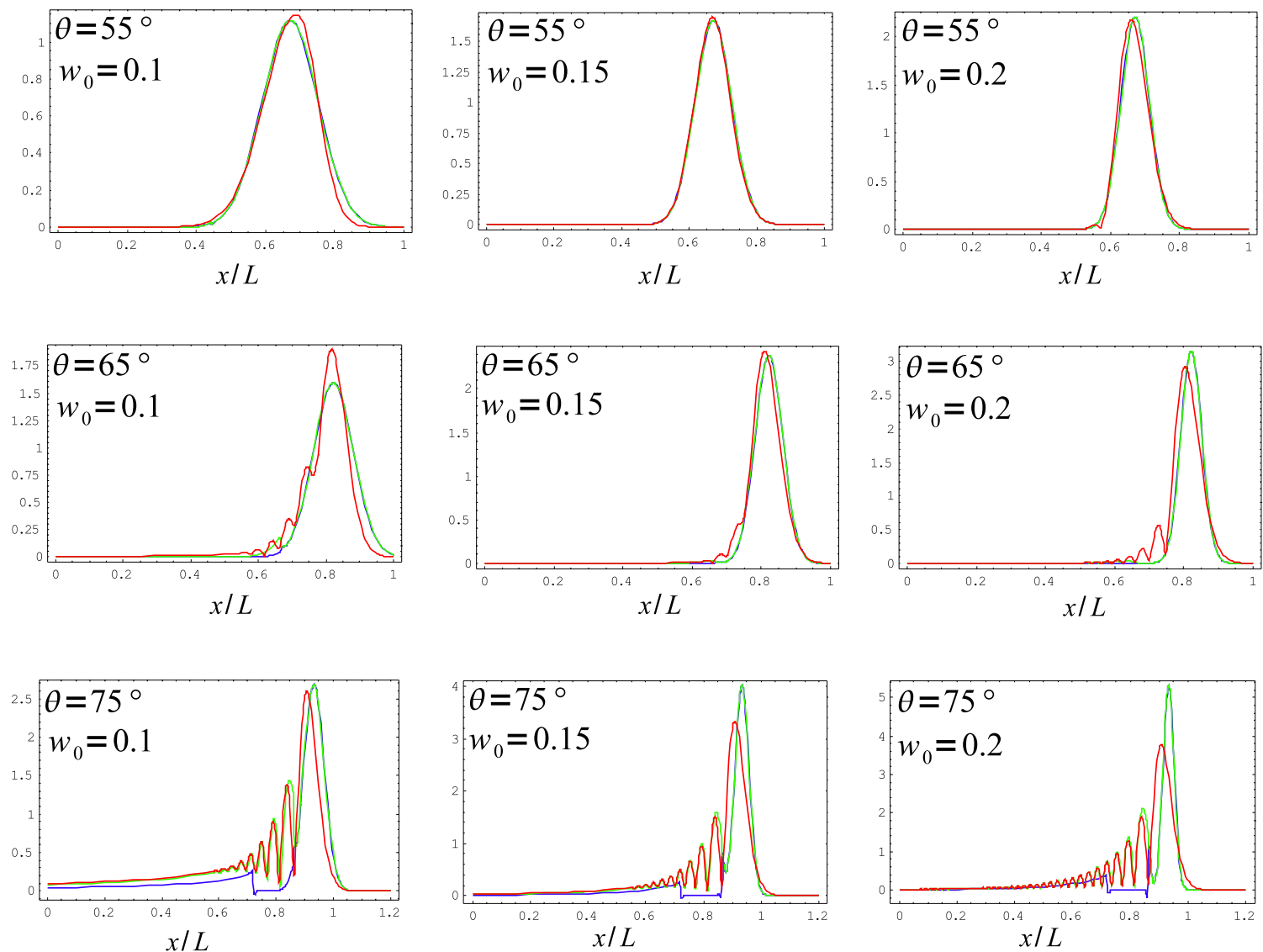

FIG. 4: (Color online) Amplitude profiles evaluated on the $x$-axis, i.e., $|u(x, 0)|$, according to the exact (red), beam tracing (blue), and generalized beam tracing (green) solutions for different beam and injection parameters.

the superposition of the wave field (14) with $\tau=\tau_{1}(\boldsymbol{q})$ and $\tau=\tau_{2}(\boldsymbol{q})$, respectively. This modification does not change the asymptotics for $\kappa \rightarrow+\infty$, but it can amount to a better approximation for finite values of $\kappa$.

We are particularly interested in the position and width of the caustic region in the $x$ coordinates which corresponds to the radial coordinate $r$, in actual tokamak geometry. For the cases under consideration, the wave field intensity attains its maximum near the $x$-axis so that the caustic can be well represented by the section $y=0$ of the corresponding solutions. Fig. 4 shows the amplitude profiles for the $y=0$ sections of the wave field, namely, $|u(x, 0)|$, for few typical cases out of the considered sample. The amplitude profiles exhibit a well marked peak near the cutoff (at $x / L=1$ ), and, depending on the angle, several secondary peaks which are the consequence of the interference of the incident and reflected waves. 
From Fig. 4 one can see that the standard beam tracing solution and its generalized form described above are superposed for low injection angles, as the geometry of the reference ray yields no multiple values for $\tau$ where the wave field is significant. For higher injection angles, the generalized beam tracing construction, indeed, matches fairly well the secondary peaks, yet near the main peak corresponding to the exact caustic it is still superposed to the standard beam tracing solution: there a single real root $\tau(\boldsymbol{q})$ is found (this can be estimated by the curvature radius $R_{\text {ray }}$ of the reference ray at the turning point) and, therefore, both beam tracing solutions yield the same amplitude profile. Increasing the injection angle, the beam tracing solution near the main peak becomes narrower than in the exact solution and shifts toward the cutoff. Qualitatively, one can also note that, for all the displayed angles, $\theta=55^{\circ}, \theta=65^{\circ}$ and $\theta=75^{\circ}$, the best matching is obtained for the intermediate value of the initial width $\alpha / \sqrt{\kappa}=0.15$ : this can be understood if we think of interference effects near the turning point that are controlled by the beam width; this is large in correspondence of both the large and the narrow initial profiles, in the latter case as a consequence of a strong diffractive broadening.

The position of the classical turning point $x_{\mathrm{tp}}^{\prime}(\theta)=\sin ^{2} \theta$ (which in the BT solution corresponds to the position of the main peak) and the width at the turning point (20) are finally compared to the the position $x^{E x}$ of the exact main peak and to the exact width, which can be estimated as

$$
w^{E x}=\frac{\sqrt{2}}{L}\left[\frac{\int_{0}^{+\infty}\left(x-x^{E x}\right)^{2}\left|u^{E x}(x, 0)\right| \mathrm{d} x}{\int_{0}^{+\infty}\left|u^{E x}(x, 0)\right| \mathrm{d} x}\right]^{\frac{1}{2}} .
$$

When such a definition is applied to the beam tracing solution, it yields exactly $w_{\text {tp }}$ and this justifies the use of Eq. (21). Other possible estimates for the width can be defined; keeping in mind reflectometry applications, the appropriate estimate of the width should be defined on the basis of the real interaction of the beam with plasma density fluctuations that are not included in our model.

The caustic position and width are plotted in Fig. 5. First, we note that, for $\theta \rightarrow 90^{\circ}$, both the exact position and width approach a limit value which is independent on the initial width. The position of the classical turning point is also independent of the initial width, and reaches the cutoff layer at $x / L=1$ for $\theta \rightarrow 90^{\circ}$. The error on the position, however, never exceeds few percent. As for the width of the caustic, the relative error can be significant even for low injection angles, when the initial beam width is too narrow. 

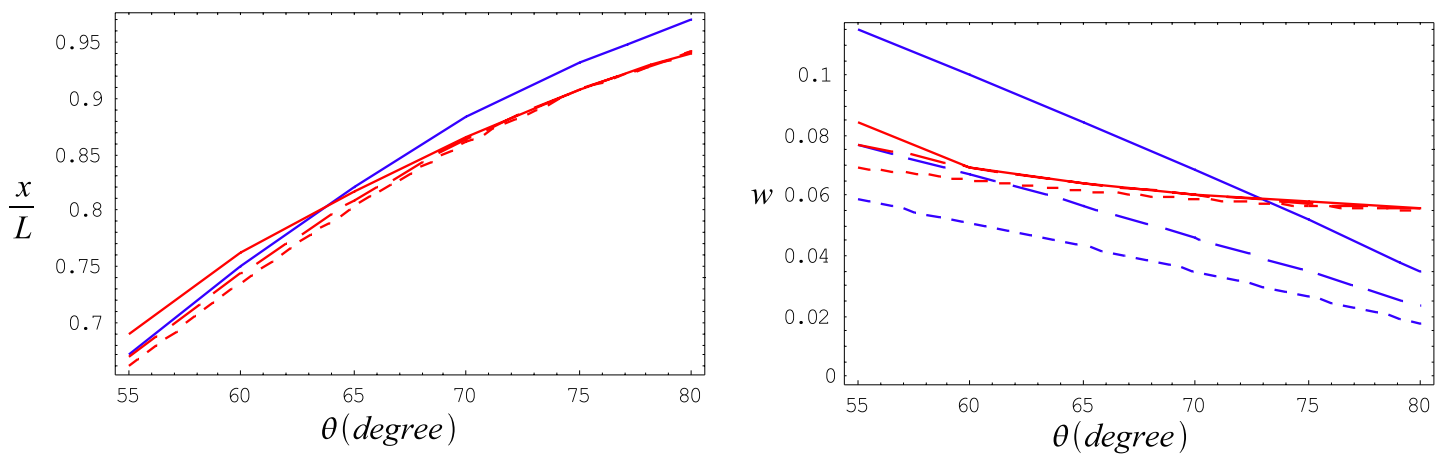

FIG. 5: (Color online) Position of the caustic (left) and its width (right) as a function of the injection angle $\theta$ for different values of the initial beam width (solid curves represent the case $\alpha=0.1 \sqrt{\kappa}$, coarse-dashed curves the case $\alpha=0.15 \sqrt{\kappa}$ and fine-dashed curves the case $\alpha=0.2 \sqrt{\kappa}$ ). Red curves correspond to the exact solution, blue curves to the BT solution. Note that the BT curves on the left are indistinguishable, since in this case the position of the caustic is given by the classical turning point, which is independent of the beam width.

Looking at the corresponding amplitude profile of Fig. 4, one can see that such a difference can be explained by the asymmetry of the exact profile which is narrower on the side of the cutoff layer, therefore, the definition (21) slightly underestimates the actual width, being unable to account for the asymmetry of the exact profile. For the cases $\alpha / \sqrt{\kappa}=0.15$ and $\alpha / \sqrt{\kappa}=0.2$ one can see that the beam tracing description yields a reliable value of the width of the main peak up to $\theta=65^{\circ}-70^{\circ}$ where the relative error is around $30 \%$.

In order to push the beam tracing method to the limits we have also considered few cases at rather low frequency $(\kappa=50)$. We find qualitatively the same behaviour with the only difference that the error on the position can be significant for high injection angles, that is, the shift of the exact caustic position with respect to the classical turning point is more significant at low frequencies. 


\section{ABSORBING HALF-PLANE}

\section{A. Exact solution}

In order to validate the BT approach for the practically significant case of ECR plasma heating we consider a solution to the Helmholtz equation (1) with the refractive index given by

$$
n^{2}(x)=\left(\frac{c k(x)}{\omega}\right)^{2}=\varepsilon+i \frac{4 \pi \sigma}{\omega}, \quad k^{2}(x)=k_{0}^{2}[1+i \gamma H(x)]
$$

with $k_{0}=\omega / c, \gamma=4 \pi \sigma / \omega$ and both $\gamma$ and $k_{0}$ do not depend on the space coordinates. $H(x)$ is the Heaviside function. For the sake of simplicity we take $\varepsilon=1$ so that the domain $x<0$ is the free space. Assuming $\gamma>0$ means that the other half-space $x>0$ describes wave absorption. The exact solution of Eqs.(1),(22) in any half-space can be expressed in any of the forms

$$
\begin{aligned}
& u(\tau, \eta)=\frac{1}{\sqrt{2 \pi}} \int_{-\infty}^{\infty} u_{\eta}\left(k_{\eta}\right) e^{i \eta k_{\eta} \pm i \tau \sqrt{k^{2}-k_{\eta}^{2}}} \mathrm{~d} k_{\eta}=\frac{1}{\sqrt{2 \pi}} \int_{-\infty}^{\infty} u_{\tau}\left(k_{\tau}\right) e^{i \tau k_{\tau} \pm i \eta \sqrt{k^{2}-k_{\tau}^{2}}} \mathrm{~d} k_{\tau} \\
& u(x, y)=\frac{1}{\sqrt{2 \pi}} \int_{-\infty}^{\infty} u_{y}\left(k_{y}\right) e^{i y k_{y} \pm i x \sqrt{k^{2}-k_{y}^{2}}} \mathrm{~d} k_{y}=\frac{1}{\sqrt{2 \pi}} \int_{-\infty}^{\infty} u_{x}\left(k_{x}\right) e^{ \pm i x k_{x}+i y \sqrt{k^{2}-k_{x}^{2}}} \mathrm{~d} k_{x}
\end{aligned}
$$

where $u_{\eta}, u_{y}$ and $u_{x}$ are arbitrary functions. Two coordinate systems are used in the representations (23)-(24). One of them $(x, y)$ is aligned with the boundary $x=0$ that separates two half-spaces in Eq. $(22)$. The second $(\tau, \eta)$ is oriented along the propagating beam as shown in Fig. 6.

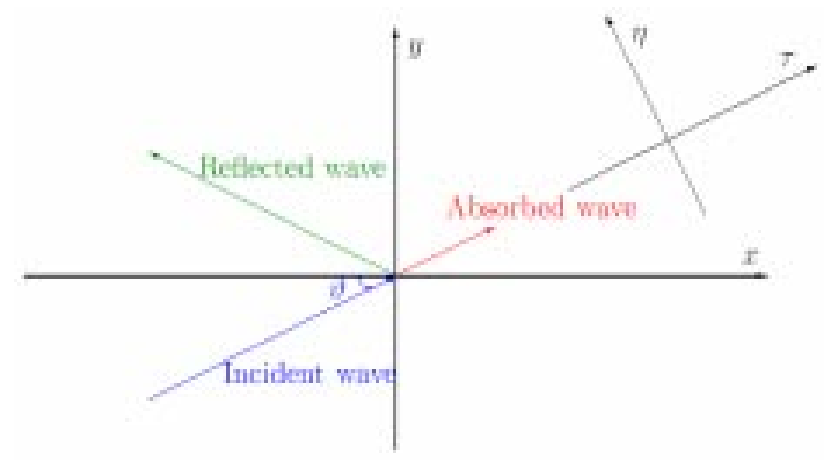

FIG. 6: (Color online) Wave geometry and the ray coordinate system.

Generally speaking, the proper branches of the square root functions in the exponents of 
Eqs. (23)-(24) have to be selected to describe a bounded solution. However, our discussion is limited to the practically important case of narrow wave beams, for which only the paraxial domain $\left|k_{\eta}\right| \ll k_{0}=\omega / c$ is essential. In this case, the contributions of short transverse and evanescent waves are exponentially small and will be discarded below. It means that the infinite integration domains in integrals above can be cut at $\left|k_{\eta}\right| \leq k_{0}$, etc. For simplicity we retain the notation of Eqs. (23)-(24) also in what follows. In addition, appropriate signs should be selected in the exponents of Eqs. (23)-(24) to describe the incident, reflected and the transmitted waves. Moreover, expressions for $u_{y}\left(k_{y}\right)$ and other amplitude functions are different on both sides of the interface surface $x=0$. Using the conditions that the solution $u(x, y)$ together with its first derivatives must be continuous at the interface $x=0$

$$
\left.u(x, y)\right|_{x=-0} ^{x=+0}=\left.u_{x}\right|_{-0} ^{+0}=\left.u_{y}\right|_{-0} ^{+0}=0
$$

we find a solution with one arbitrary function $u_{0, y}\left(k_{y}\right)$

$$
u(x, y)=\frac{1}{\sqrt{2 \pi}} \begin{cases}\int_{-\infty}^{\infty} u_{0, y}\left(k_{y}\right) e^{i y k_{y}}\left\{e^{i x \sqrt{k_{0}^{2}-k_{y}^{2}}}+\left(F\left(k_{y}\right)-1\right) e^{-i x \sqrt{k_{0}^{2}-k_{y}^{2}}}\right\} \mathrm{d} k_{y}, & \text { if } x<0, \\ \int_{-\infty}^{\infty} u_{0, y}\left(k_{y}\right) F\left(k_{y}\right) e^{i y k_{y}+i x \sqrt{k_{0}^{2}-k_{y}^{2}+i k_{0}^{2} \gamma}} \mathrm{d} k_{y}, & \text { if } x>0 .\end{cases}
$$

where

$$
F\left(k_{y}\right)=\frac{2}{1+\sqrt{1+i B}}, \quad B=\frac{k_{0}^{2} \gamma}{k_{0}^{2}-k_{y}^{2}}=\frac{\gamma}{\cos ^{2} \vartheta}
$$

\section{B. BT solution}

The exact solution (26) will be compared with the fundamental mode of the BT solution[34]

$$
u^{B T}(\boldsymbol{r})=u^{B T}(\tau, \eta)=\frac{A(\tau)}{\sqrt{w} \sqrt[4]{\pi}} e^{i k_{0} \tau-\frac{\eta^{2}}{2 w^{2}}(1-i \theta)-\frac{i}{2} \arctan \theta}
$$

where $\theta(\tau)=\tau /\left(k_{0} w_{0}^{2}\right), w(\tau)=w_{0} \sqrt{1+\theta^{2}(\tau)}=\sqrt{w_{0}^{2}+\tau^{2} /\left(k_{0} w_{0}\right)^{2}}$. It is assumed additionally that, in the absence of absorption, the minimum wave beam waist is given by $w_{0}$ and located at the position $\tau=0$ along the reference ray. In coordinates $(x, y)$ the same point is given by $\left(x_{0}, y_{0}\right)$ (in the numerical calculations that follow, the position of the beam waist 
$\tau=0$ is assumed to coincide with the point $\left(x_{0}=0, y_{0}=0\right)$ where the beam crosses the absorbing half plane, cf. Fig. 7, since an EC heating system is usually designed to achieve the best focusing in the absorption region). Note that, due to the ordering employed during the construction of the BT solution, the functions $\theta(\tau), w(\tau)$ and $A(\tau)$ are defined along the reference ray only, i.e. at $\eta=0$. The BT solution (28) describes a wave beam with the the group velocity directed along $\mathbf{e}_{\tau}=\cos \vartheta \mathbf{e}_{x}+\sin \vartheta \mathbf{e}_{y}$. The beam is localised around the reference ray $y-y_{0}=\left(x-x_{0}\right) \tan \vartheta$, has the width $w(\tau)$ and the amplitude $A(\tau)$. By integrating Eq. (28) we find

$$
W(\tau)=\int_{-\infty}^{\infty}\left|u^{B T}(\tau, \eta)\right|^{2} \mathrm{~d} \eta=\frac{|A(\tau)|^{2}}{w \sqrt{\pi}} \int_{-\infty}^{\infty} e^{-\frac{\eta^{2}}{w^{2}}} \mathrm{~d} \eta=|A(\tau)|^{2}
$$

and note that the absolute value $|A|$ of the amplitude is directly related to the linear density of the wave energy along the beam $W$. It remains to determine any of those quantities. The standard way of doing this would be to solve the transport equation that is attributed to the second order of asymptotic expansion in powers of the small parameter $\kappa^{-1 / 2}[11]$.

To get more insight into the physics properties of the solution we substitute the BT solution (28) in the energy conservation law (4)

$$
\operatorname{div}\left[\left|u^{B T}\right|^{2}\left(\nabla \tau+\frac{\tau \eta \nabla \eta}{w_{0}^{2} w^{2}(\tau)}\right)\right]+k_{0} \gamma(\mathbf{r})\left|u^{B T}\right|^{2}=0 .
$$

The second term in brackets describes the local flux across the wave beam axis, i. e. across direction of the group velocity. Because of the multiplier $\eta$ it should be scaled as $\kappa^{-1 / 2}$ and therefore contributes to higher order corrections only. This term describes a transversal flux of wave energy. Strictly following the procedure of the asymptotic expansion [11] one has to discard the discussed term in spite of its clear physical meaning. This would represent the fact that the paraxial expansion considers the Gaussian wave beam modes as indivisible physics objects (approximate eigenmodes of the parabolic equation). In this respect the beam tracing description is similar to ray tracing: the energy flux is directed along the vector of the group velocity $\mathbf{e}_{\tau}$ exclusively. In other words, although an infinitely thin ray is replaced with a finite-width beam its internal structure still remains beyond the scope of the adopted approximation.

The next simplification of the beam tracing technique results in replacing the twodimensional conservation law (30) by an ordinary differential transport equation for the 
wave amplitude $|A|$. The transition is equivalent to integrating the local relation (30) over any unbounded curve in the $(x, y)$ plane. The result of integration is an ordinary differential equation for the amplitude $A(\tau)$. Its solution does not depend on the shape of the curve. To be more precise, the dependence on the integration path shows up in higher orders of the asymptotic expansion.

In this paper, we want to explore the (experimentally relevant) situation in which absorption is not small, i.e. cannot be scaled as $\gamma=\mathcal{O}\left(\kappa^{-1}\right)$. In this case, one cannot claim that the result of integration of the second term in Eq. (30) is path independent. Moreover, this integral cannot be calculated analytically, and to obtain the field amplitude $A(\tau)$ one has to solve an integral equation. In order to overcome this difficulty, we select the contours of integration in Eq. (30) as lines of $\gamma(\mathbf{r})=$ Const. Then $\gamma(\tau)$ can be evaluated on the reference ray and taken out of the integrand. The resulting equation again acquires the form of an rdinary differential equation for the field amplitude that can be easily solved. The energy conservation law (30) averaged along the contour levels of absorption will be satisfied.

Before moving to a particular example we summarize the adopted assumptions. First of all, it is assumed that for this problem the large parameter $\kappa$ can be introduced and that the usual BT scaling is applicable. Then the beam-like structure of the solution is mainly retained even in the case of finite or rapidly varying absorption. This seems to be reasonable as long as the eigenfunctions are determined by the Hermitian part the original wave equation (1) and until the absorption exceeds the level of $\gamma=\mathcal{O}\left(\kappa^{-1 / 2}\right)$. Basing on this property we employ the energy conservation law and derive a special form of the amplitude transport equation. By this replacement we give to the energy conservation law (that is an exact relation) more credit than to the standard transport equation (that has been derived as the second order of the asymptotic expansion). This way of calculating the wave field can be easily extended to arbitrary geometry and is implemented in the beam tracing code TORBEAM [15].

In order to illustrate the aforesaid and to obtain $|A(\tau)|$ we apply the integral $\int_{-\infty}^{\infty} \mathrm{d} y$ to Eq. (30)

$$
\frac{\mathrm{d}}{\mathrm{d} x} W\left(\frac{x-x_{0}}{\cos \vartheta}\right)+\frac{\gamma k_{0} H(x)}{\cos \vartheta} W\left(\frac{x-x_{0}}{\cos \vartheta}\right)=0,
$$

where $\tau$ was replaced by $\tau=\left(x-x_{0}\right) / \cos \vartheta$ and the relation $\mathrm{d} \eta=\mathrm{d} y \cos \vartheta$ has been taken 
into account. Solution of Eq. (31) reads

$$
W(x)=W_{0} e^{-\gamma k_{0} x H(x) / \cos \vartheta}
$$

and with account of Eq. (29) defines the amplitude evolution along the wave beam

$$
|A(\tau)|=A_{0} \begin{cases}1, & \text { if } \quad \tau<\tau_{0} \\ e^{-\gamma k_{0}\left(\tau-\tau_{0}\right)}, & \text { if } \quad \tau \geq \tau_{0}\end{cases}
$$

where $\tau_{0}=-x_{0} / \cos \vartheta, A_{0}=\sqrt{W_{0}}$. The BT solution is now completely defined. As expected, the energy flux through the plane $x=$ Const remains unchanged until the beam enters the absorption zone $x \geq 0$. Consider again the energy conservation law in the form Eq. (30) and observe that the second term describes the power deposition density. This suggests to introduce two quantities

$$
P(x)=H(x) \int_{-\infty}^{\infty}|u(x, y)|^{2} \mathrm{~d} y, \quad P(y)=\int_{-\infty}^{\infty}|u(x, y)|^{2} H(x) \mathrm{d} x,
$$

that are proportional to the line density of the power absorption. The two quantities (34) can be associated with the power density deposition within a flux surface of a tokamak and will be discussed in the next Section. The first of them is proportional to the power density $W(\tau)$ if $x \geq 0$

\section{Comparison of BT and exact solutions}

In order to evaluate the error introduced by the approximate character of the BT solution we define the arbitrary function $u_{0, y}\left(k_{y}\right)$ in Eq. (26) by the condition

$$
u_{0, y}\left(k_{y}\right) \mathrm{d} k_{y}=\frac{A \sqrt{w_{0}}}{\sqrt[4]{\pi}} \exp \left(\tau_{0} \sqrt{k_{0}^{2}-k_{\eta}^{2}}-\frac{1}{2} w_{0}^{2} k_{\eta}^{2}\right) \mathrm{d} k_{\eta},
$$

where

$$
k_{\eta}=k_{y} \cos \vartheta-\sin \vartheta \sqrt{k_{0}^{2}-k_{y}^{2}}, \quad \mathrm{~d} k_{\eta}=\left(\cos \vartheta+\frac{k_{y} \sin \vartheta}{\sqrt{k_{0}^{2}-k_{y}^{2}}}\right) \mathrm{d} k_{y} .
$$

This condition ensures that the exact (26) and the asymptotic (28) solutions under consideration coincide at $k_{0} \rightarrow \infty$.

As the first illustration we show the contour levels of the absolute value for the exact solution in Fig. 7a. Unlike all other figures the coordinates are inverted here $(x, y) \rightarrow$ 

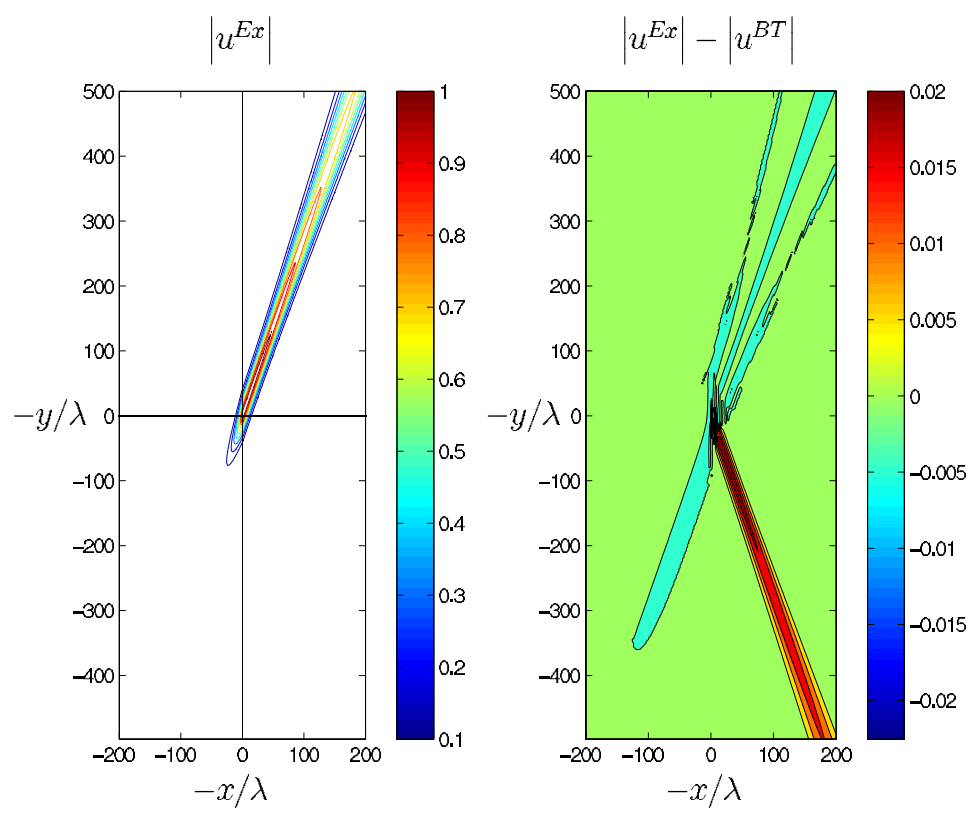

FIG. 7: (Color online) Contour levels of the wave field amplitude. Parameters are selected to fit the ITER ECRH system: $f=170 \mathrm{GHz}, w_{0}=1.48 \mathrm{~cm}, \vartheta=70^{\circ}, \gamma=0.01$ [note that the beam width as usually defined in ITER documents is by a factor $\sqrt{2}$ larger than the beam width defined in Eq. (28)]. The coordinates are inverted to represent the ITER geometry. (a) Contours of $\left|u^{e x}(x, y)\right|$, (b) contours of $\left|u^{e x}(x, y)\right|-\left|u^{B T}(x, y)\right|$.

$(-x,-y)$ to draw an analogy with the upper-launcher geometry of ITER. First of all, we note that for zero absorption the wave field would be quasi-symmetric with respect to the point $x=y=0$. Because of the large factor $k_{0}=35.6$ in the second term of Eq. (30) even rather small $\gamma=0.01$ gives a noticeable exponential decay in the 3rd quadrant (the choice $\gamma=0.01$ gives a good estimate of the absorption coefficient for a typical ITER scenario [33]).

In order to evaluate the error of the BT solution in the next Fig. $7 \mathrm{~b}$ we plotted the residual function $\left|u^{e x}(x, y)\right|-\left|u^{B T}(x, y)\right|$. In this plot, the resolution is higher than in Fig. 7 by more than two orders of magnitude. This allows one to see the difference between exact and asymptotic solutions. It follows that the overall accuracy of the BT solution is better than $1 \%$. In particular, it remains at absolutely negligible level in the first quadrant where the incident wave is localized. In this domain the BT approach is justified up to the third order of the asymptotic expansion. The next observation is the existence of a reflected wave that is fully discarded by the BT solution. In Fig. $7 \mathrm{~b}$ the reflected wave is seen as a beam 


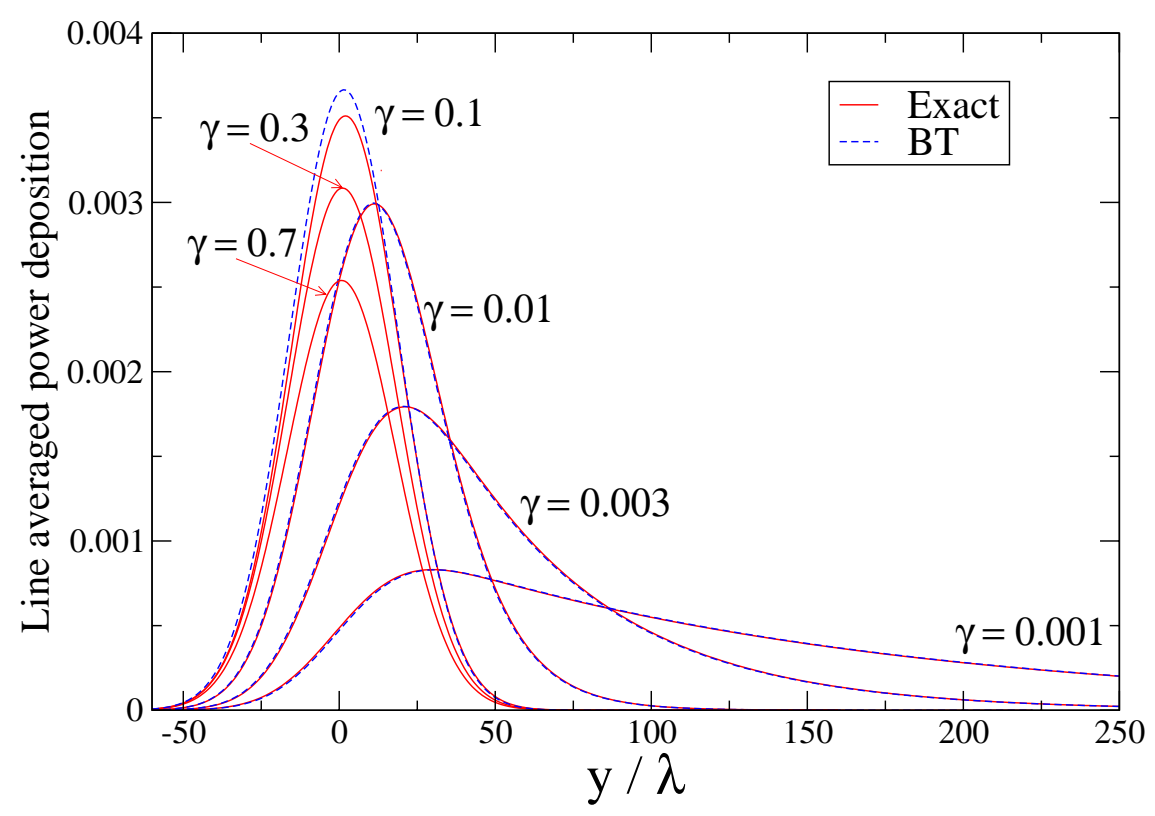

FIG. 8: (Color online) Function $P(y)$ for different values of $\gamma$. Solid red lines correspond to the exact solution, dashed blue lines to the BT solution.

in the fourth quadrant. Another distinction between the two solutions is observed in the absorption zone. It is not surprising that errors are of the same order in zones of reflected and absorbed waves because they have the same origin and are caused by the discontinuity in $n^{2}$ at $x=0$. The field behaviour in the absorption zone is of our main interest because according to Eq. (34) the quantity $|u(x>0, y)|^{2}$ directly describes the power deposition profile.

If the beam hits the absorption zone in the mid-plane of a tokamak, then averaging over the flux surfaces can be represented by the averaging over the plane $y=$ Const in our simplified geometry, i.e. described by the function $P(x)$, Eq.(34). Apart from a multiplier, this function coincides with $W(x)$, that can be easily calculated by means of the simple formula Eq. (32). If the absorption zone is shifted up or down with respect to the midplane, then averaging over the flux surfaces corresponds to averaging over the plane $x=$ Const, i.e. described by the function $P(y)$. This function is shown in Fig. 8 for different values of $\gamma$.

As seen, for small $\gamma$, the profiles have long tails that are strongly extended to the large values of $y$. With increasing $\gamma$ the deposition profile becomes more localized and its maximum moves closer to $y=0$. Up to value $\gamma=0.1$ exact and BT profiles are practically indistinguishable (this is found to be the case also when the beam width is varied within a 


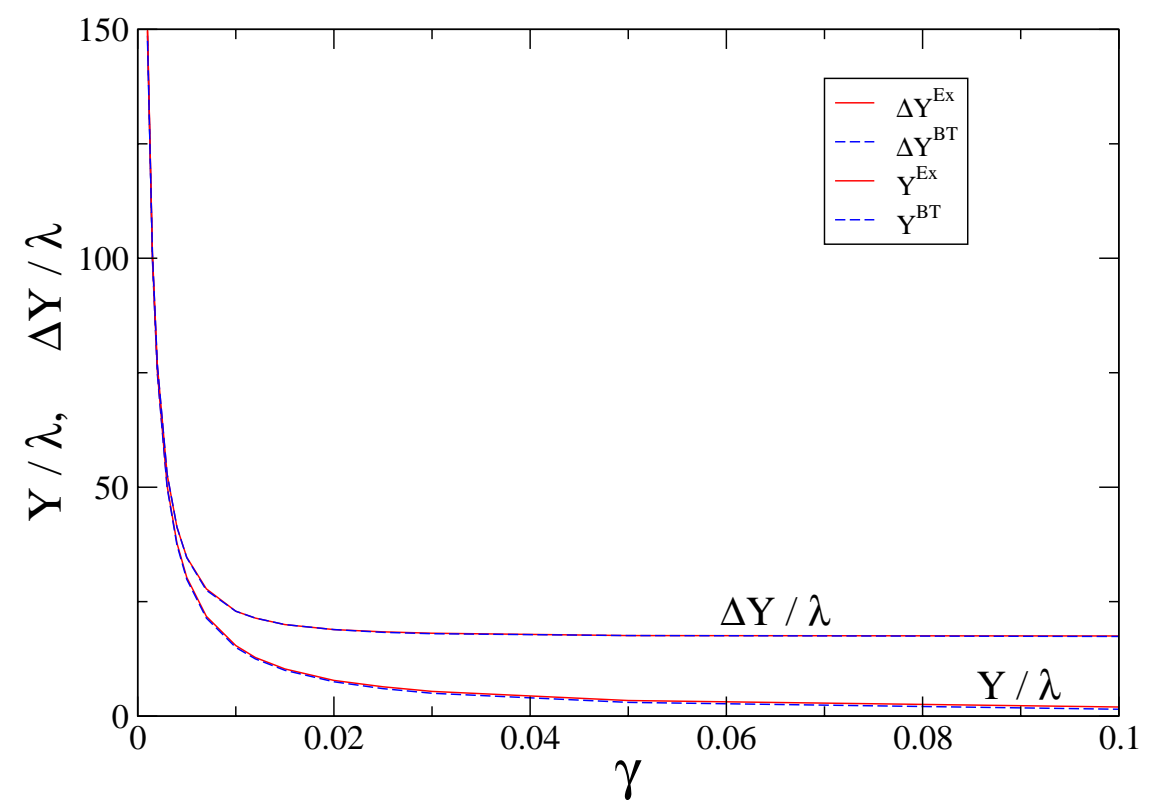

FIG. 9: (Color online) Moments of the function $P(y)$ versus $\gamma$. Solid red lines correspond to the exact solution, dashed blue lines to the BT solution.

realistic range of values). If the absorption ocefficient is increased above 0.1 , both profiles acquire Gaussian form but start to diverge. This happens because the absorption as described by the exact solution starts to decrease due to growth of the reflection. The beam tracing solution does not include reflection and therefore the profile $P(y)$ does not change with further increase in $\gamma>0.1$.

These properties are summarized in Fig. 9, where the function $P(y)$ is represented by its moments. As discussed, the distribution width $\Delta Y=\sqrt{\left\langle(y-Y)^{2}\right\rangle}$ and its centre $Y=\langle y\rangle$, rapidly reduce from infinity at low absorption to several wavelengths as $\gamma$ reaches values of order 0.01. However, at this value of $\gamma$ the distribution width $\Delta Y$ starts to saturate while $Y$ continuously decreases. As long as at $\gamma \geq 0.01$ the distribution profile becomes Gaussian its shape is fully described by these two moments. This behaviour can be easily understood. Indeed, at large $\gamma$ the wave absorption is strong and the absorbed power is concentrated near the plane $x=0$. Its shape must be Gaussian because the wave field is continuous and it is Gaussian at $x<0$. Moreover, the width of this Gaussian is defined by a longitudinal (with respect to the group velocity) projection of the transversal width $w(\tau)$ to the plane $x=0$. This gives the estimate for the asymptotic values of the width $\Delta Y \rightarrow w_{0} /(\sqrt{2} \cos \vartheta)$ and of the shift $Y \rightarrow y_{0}-x_{0} \tan \vartheta$, the latter in our case being zero. 


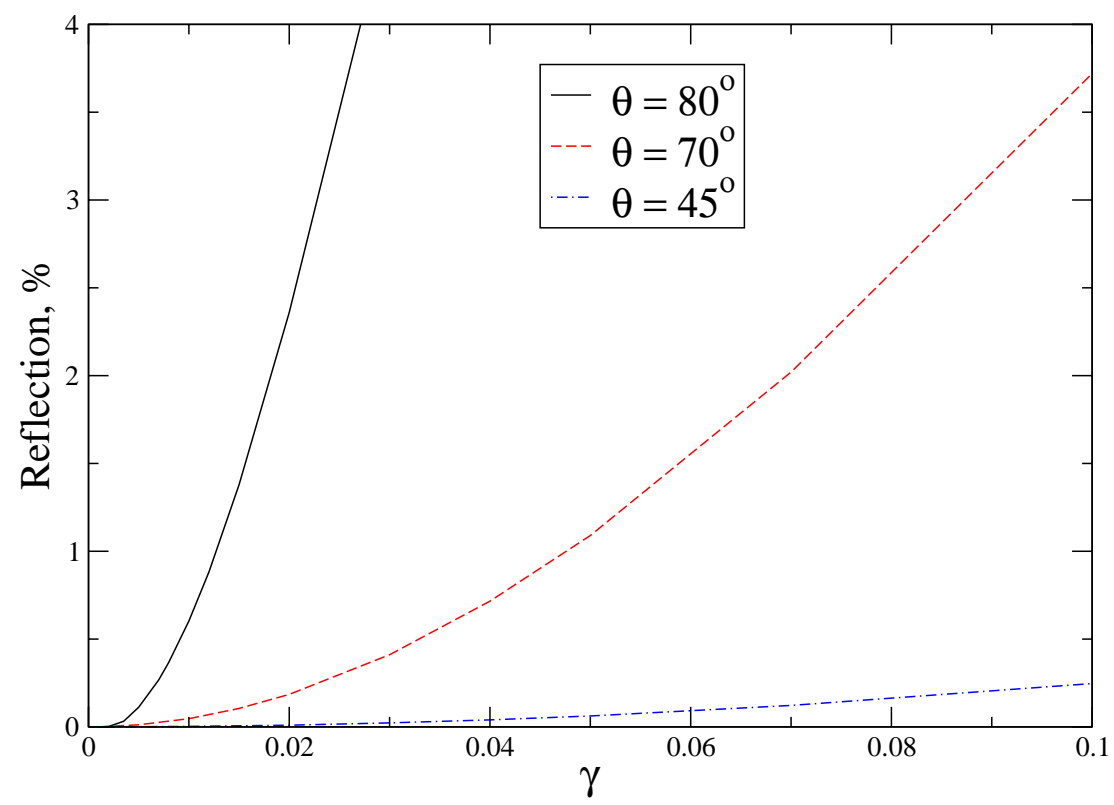

FIG. 10: (Color online) Reflection coefficient as a function of $\gamma$ for different launch angles.

It follows that the BT solution can serve as a very good approximation to the exact one unless the reflection becomes significant. Moreover, it should be emphasized that in all respects involving absorption our case of discontinuous $n^{2}(\boldsymbol{r})$ is the most difficult for an asymptotic treatment and one can expect here the least accuracy; in particular, this case overestimates reflection. Inverting this statement we conclude that applying the BT technique to the real plasma one can expect better agreement than in the case considered here. In order to assess the limits of the BT applicability to EC waves in a tokamak plasmas and thus a relevance of our study we compare our results with the realistic numerical modelling [15], [21]. As a characteristic measure for such a comparison one can select the wave decay length which in tokamaks is usually a few centimeters. This value corresponds to $\gamma \approx 0.01 \ll 1$ and is far below the limit of $\gamma=0.1$ where some discrepancies can be observed. In the range $\gamma \approx 0.01$ all distinctions between the exact and BT solutions are far below the required accuracy of simulations.

We have still to discuss how this limit in $\gamma$ depends on the beam launching conditions. If we consider the reflection coefficient $R$ as an indicator of BT invalidity, then inspection of Eq. (27) shows that the wave reflection depends on the combination $\gamma / \cos ^{2} \vartheta$ of the two parameters $\gamma$ and $\vartheta$. Influence of the angle of incidence $\vartheta$ is shown in Fig. 10. It is seen that the reflection has to be taken into account when the wave is launched very close to the 
cyclotron resonance position $\omega=\omega_{B e}$ and propagates nearly vertically. Even for $\vartheta=80^{\circ}$ and for $\gamma=0.01$ the reflection coefficient is $R=0.6 \%$. However, this case is unrealistic and has never been considered as an option. The upper-launcher geometry foreseen for ITER corresponds to maximum injection angle below $\vartheta=70^{\circ}$. In this case $R=0.05 \%$. Already this value is overestimated and has to be reduced if the smooth behaviour of the absorption coefficient is taken into account. We conclude that for typical tokamak parameters all distinctions between the exact and BT solutions in this range of absorption rates are far below the significance limit for the problem of interest.

\section{CONCLUDING REMARKS}

The two wave-propagation problems considered in this paper, namely the reflection of a wave beam by a cutoff layer and the oblique propagation in the presence of an absorbing half-plane, represent two test cases for which asymptotic techniques like paraxial WKB beam tracing are pushed to their limits of applicability. In the first situation, the beam tracing model does not reproduce the diffractive pattern originating from a significant overlap of the injected and reflected wave field. In the second, strong absorption could lead to a breakdown of the pWKB solution, which does not account correctly for changes occurring on the scale of the beam width. Moreover, for high values of the absorption coefficient, a non-negligible fraction of the injected power (not described by beam tracing) could be reflected as the beam impinges onto the absorption layer. On the other hand, since the conditions mentioned above are often met in the experiments, the use of asymptotic techniques is highly desirable if they can be shown to represent a reliable alternative to CPU-intensive full-wave computations.

The results presented in Sec. III-IV show that, for realistic parameters, the pWKB approach is quite robust. In particular, for the case of reflection from a cutoff layer, the location of the caustic is well reproduced by the beam tracing solution, the difference with respect to the exact position being of the order of a few percent for experimentally relevant injection parameters. If the injection is not too close to perpendicular $\left(\theta \lesssim 70^{\circ}\right)$, moreover, also the width of the caustic can be estimated employing paraxial beam tracing, the relative error being in this case of the order of 30\%, for instance, for parameters corresponding to the typical setup of Doppler reflectometry at ASDEX Upgrade. Also in the case of oblique injection onto an absorbing half-plane, deviation of the BT power-absorption profile with 
respect to the exact one is found only at very high values of the absorption coefficient and is basically due to the power reflected at the interface with the absorbing region. Of course, our model does not entirely reproduce the typical conditions of oblique ECRH in ITER. The real injection geometry (where a nonzero toroidal injection angle is foreseen) is not included. Moreover, the absorption coefficient has been assumed to be constant. These approximations, however, do not seem to impair the capability of our approach to give a good qualitative description of the basic features of the process. In particular, the assumption of a constant absorption coefficient represents a worst-case scenario for an estimate of the reflected power at the resonance, so that our finding of negligible reflection should be verified in ITER.

The analysis presented in this paper has been partly motivated by a recent work of Balakin et al. [25, 26]. In particular, a statement about the applicability of the pWKB description to the case of beam reflection by a linear layer has been shown here to be clearly incorrect. Non-paraxial effects for the power-absorption profile, which have been found to be negligible within our model, deserve a careful investigation under more realistic ITER conditions. The "quasi-optical" description put forward in Ref. [26] (and references therein) does not resolve the issue because it relies on heuristic considerations rather than on a rigorous asymptotic analysis, an accurate benchmarking of the model should be addressed. Although a thorough analysis of such a description is far beyond the aim of this paper, here we mention a few points that should be carefully scrutinized. The derivation of the relevant equations of the "quasioptical" method [26] consists in two main steps: (i) the derivation of the scalar equation for the considered wave mode and (ii) the approximation of such an equation by an evolutionary partial differential equation. In (i), one has to put a system of (pseudo-)differential operators into diagonal form: this is a well known problem, cf. Ref. [32] for a quantum mechanical application, and such a diagonalization procedure can be rigorously carried out under rather restrictive conditions, e.g., for weakly non-Hermitian and slowly varying media, that break down exactly at the resonance layer where the absorption coefficient varies significantly in a short range. As for (ii), the derivation of the evolutionary equation is carried out on the basis of heuristic modelling, and such a procedure do require further work toward a precise asymptotic theory controlled by a well-defined explicit parameter.

\section{Acknowledgments}


Many useful discussions with R. Bilato, M. Brambilla and G. Conway are gratefully acknowledged. The work of O.M. has been supported by the Foundation Blanceflor Boncompagni Ludovisi.

\section{APPENDIX A: LOCAL COORDINATE SYSTEMS AND BEAM PARAMETERS}

In this appendix we give a mere summary of technical details about the construction of the beam tracing solution and the encoding of the information on the beam width and curvature of the phase fronts in the complex matrix $\Psi_{\alpha \beta}$. We refer to the original papers on the beam tracing method $[10,11]$ for the proofs. Let us discuss these issues in full generality, i.e., in the three-dimensional space, and then specialize the results to the cases under consideration.

In the beam tracing method one takes advantage of local coordinates constructed around the reference ray. The first coordinate is always given by the parameter $\tau$ along the reference ray; then the system is completed by adding two transverse coordinates $\left(\eta^{1}, \eta^{2}\right)$ defined as follows. In each point $\boldsymbol{q}(\tau)$ one chooses a plane which represents a beam cross-section; let it be spanned by two unit vectors $\left\{\boldsymbol{e}_{1}(\tau), \boldsymbol{e}_{2}(\tau)\right\}$. Then local coordinates are defined by

$$
q^{\alpha}=q^{\alpha}(\tau)+e_{i}^{\alpha}(\tau) \eta^{i}
$$

where the sum over repeated indices is implied with Greek indices for quantities in the physical space and Latin indices for quantities on the transverse cross-sections. Mathematically, the only required condition is that $\left\{\mathrm{d} \boldsymbol{q}(\tau) / \mathrm{d} \tau, \boldsymbol{e}_{1}(\tau), \boldsymbol{e}_{2}(\tau)\right\}$ must constitute a set of three linearly independent vectors throughout the reference ray; this is enough for the Jacobian of the transformation to be non-zero on the reference ray, and, thus for $\left(\tau, \eta^{1}, \eta^{2}\right\}$ to constitute well-defined coordinates in a small neighbourhood of the reference ray. In applications, however, we need the coordinate system, at least, in a whole neighbourhood of radius $w$ which requires that the selected beam cross-section should not be too far from the normal cross section, i.e., vectors $\boldsymbol{e}_{i}(\tau)$ should be nearly orthogonal to the group velocity $\mathrm{d} \boldsymbol{q} / \mathrm{d} \tau$.

Once the local coordinates have been fixed, the calculation of the function $\tau=\tau(\boldsymbol{q})$ in the beam tracing solution (14) requires just the inversion of (A1) which can be carried out by algebraic means.

It is clear, however, that, there are many possible choice of such coordinate systems, 
yet one can prove that the difference of the corresponding beam tracing solutions is of order $O(1 / \sqrt{\kappa})$. Among all possible coordinate systems of the form (A1), a special one, beam coordinates, can be identified, which is related to the solution of the matrix Riccati equation (12). For its definition, we need to recall few mathematical properties of equations (12) and (13):

(i) the constraints (13) are consistent with the Riccati equation (12), i.e., if (13) is satisfied at $\tau=0$, then it is satisfied for any $\tau \geq 0$;

(ii) a symmetric initial datum yields a symmetric solution;

(iii) if $\phi_{\alpha \beta}(0)$ is positive (resp., non-negative) definite, then $\phi_{\alpha \beta}(\tau)$ is positive (resp., nonnegative) definite for any $\tau \geq 0$;

(iv) let $e_{\tau}^{\alpha}=\left|\frac{\mathrm{d} q}{\mathrm{~d} \tau}\right|^{-1} \frac{\mathrm{d} q^{\alpha}}{\mathrm{d} \tau}$ be the unit tangent vector to the reference ray and $e_{i}^{\alpha}$ be two unit vectors normal to it; then (13) determines the components $\tilde{\Psi}_{\tau \tau}=e_{\tau}^{\alpha} e_{\tau}^{\beta} \Psi_{\alpha \beta}$ and $\tilde{\Psi}_{\tau i}=\tilde{\Psi}_{i \tau}=e_{\tau}^{\alpha} e_{i}^{\beta} \Psi_{\alpha \beta}$, the only remaining unknown components being $\tilde{\Psi}_{i j}=e_{i}^{\alpha} e_{j}^{\beta} \Psi_{\alpha \beta}$.

Due to their physical meaning, cf., comments after equation (14), we are particularly interested in symmetric solutions with $\phi_{\alpha \beta}$ non-negative definite. Indeed, the Gaussian envelope of the beam is $\phi=\frac{1}{2} \phi_{\alpha \beta}\left(q^{\alpha}-q^{\alpha}(\tau)\right)\left(q^{\alpha}-q^{\alpha}(\tau)\right)$ and since the vector $\boldsymbol{q}-\boldsymbol{q}(\tau)$ belongs to a cross-section that amounts to $\phi=\tilde{\phi}_{i j} \eta^{i} \eta^{j}$, where $\tilde{\phi}_{i j}=\operatorname{Im} \tilde{\Psi}_{i j}$, and this must be a positive definite symmetric quadratic form. Moreover, we can always select $\boldsymbol{e}_{1}$ and $\boldsymbol{e}_{2}$ so that $\tilde{\phi}_{i j}$ is diagonal and this defines the beam coordinates. In such a reference frame $\phi_{\alpha \beta}$ is diagonal with eigenvalues $\left\{0,2\left(\kappa w_{1}^{2}(\tau)\right)^{-1}, 2\left(\kappa w_{2}^{2}(\tau)\right)^{-1}\right\}$ where $w_{i}(\tau)$ are the two normal width of the beam cross-section (normalized to the scale length $L$ ).

We conclude that the beam coordinates are those for which the information on the beam width is made explicit, i.e., beam coordinates follow the rotation of the beam throughout the propagation. In addition, this also gives the rule to compute the normal beam width from the matrix $\phi_{\alpha \beta}$.

Analogously one could as well select $\boldsymbol{e}_{1}$ and $\boldsymbol{e}_{2}$ so that the real part $\tilde{N}_{i j}=\operatorname{Re} \tilde{\Psi}_{i j}$ is diagonal and the eigenvalues are $1 / R_{1}(\tau)$ and $1 / R_{2}(\tau)$, with $R_{1}(\tau)$ and $R_{2}(\tau)$ being the two normal radii of curvature of the phase front (a two-dimensional surface). This provides a way to compute the radii of curvatures from the matrix $N_{\alpha \beta}$, yet the resulting system of coordinates is somewhat less fundamental as it does not diagonalize the whole matrix $N_{\alpha \beta}$. 
For the two-dimensional models considered in this paper, the algebra of local reference frame simplifies considerably. Equation (A1) reads

$$
\left\{\begin{array}{l}
x^{\prime}=x^{\prime}(\tau)+e_{x}(\tau) \eta \\
y^{\prime}=y^{\prime}(\tau)+e_{y}(\tau) \eta
\end{array}\right.
$$

where $\left(x^{\prime}, y^{\prime}\right)$ are Cartesian coordinates normalized to $L$ and we have only one transverse direction given by $\boldsymbol{e}(\tau)=\left(e_{x}(\tau), e_{y}(\tau)\right)$. The calculation of the function $\tau(\boldsymbol{q})$ can be carried out by multiplying the first equation in (A2) by $e_{y}$ and the second one by $e_{x}$ and subtracting the results with the result,

$$
e_{y}(\tau)\left(x^{\prime}-x^{\prime}(\tau)\right)-e_{x}(\tau)\left(y^{\prime}-y^{\prime}(\tau)\right)=0
$$

With the choices of Sec. IIIC, equation (A3) amounts to a cubic equation for $\tau$ which, for $\left(x^{\prime}, y^{\prime}\right)$ fixed, can have either one real root (plus two complex roots) or three real roots. In the first case $\tau$ is just given by the only real root, while in the second case, we pick the root for which the distance $|\boldsymbol{q}-\boldsymbol{q}(\tau)|$ is minimum; that yields a smooth local coordinate system around the reference ray. The generalized beam tracing solution addressed in Sec. IIIC takes into account the existence of multiple real roots. Specifically, where three real roots $\tau_{1}, \tau_{2}$, and $\tau_{3}$ are found with, say, $\tau_{1}<\tau_{2}<\tau_{3}$, then $\tau_{1}$ corresponds to the incoming branch of the beam, while $\tau_{3}$ corresponds to the reflected branch; the generalized beam tracing solution is obtained as the sum of two wave-objects of the form (14) obtained with $\tau=\tau_{1}$ and $\tau=\tau_{3}$, respectively. By the analysis of the discriminant of the cubic equation (A3) one can see that around the classical turning point only a single real root is found, thus, the generalized and the standard beam tracing solutions should agree near the turning point as obtained numerically.

As for the calculation of the normal beam width and phase-front curvature in the twodimensional case, it is enough to compute beam coordinates due to the low dimensionality of the problem; those are given by the unit transverse vector $\boldsymbol{e} \propto\left(-\mathrm{d} y^{\prime}(\tau) / \mathrm{d} \tau, \mathrm{d} x^{\prime}(\tau) / \mathrm{d} \tau\right)$. Then, $\operatorname{Re} \tilde{\Psi}_{22}=e^{\alpha} e^{\beta} N_{\alpha \beta}=1 / R$, where $R=R(\tau)$ is the radius of curvature (normalized to $L)$ of the phase front at $\boldsymbol{q}(\tau)$; on the other hand, $\operatorname{Im} \tilde{\Psi}_{22}=e^{\alpha} e^{\beta} \phi_{\alpha \beta}=2 /(\kappa w)^{2}$, where $w(\tau)$ is the $e^{-1}$-width (normalized to $L$ ) of the beam cross-section at $\boldsymbol{q}(\tau)$. At $\tau=0$ the matrix $\Psi_{\alpha \beta}$ depends only on the parameter $\mu=2 / \alpha^{2}-i \beta$ defined in (15), and, after some algebra, the foregoing relations yield equations (16) that relates the two parameters $\alpha$ and $\beta$ to the 
initial beam width $w(0)$ and phase-front curvature $1 / R(0)$ both normalized to $L$.

[1] L. D. Landau, E. M. Lifshitz, The Classical Theory of Fields, 4th revised ed., Pergamon Press, 1987.

[2] Yu. A. Kravtsov, Yu. I. Orlov, Geometrical Optics of Inhomogeneous Media, Springer Series on Wave Phenomena, Vol. 6, Springer Verlag, 1990.

[3] I. B. Bernstein, Phys. Fluids 18, 320 (1975).

[4] S. Choudhary, L. B. Felsen, IEEE Trans. Antennas Propag. AP-21, 827 (1973).

[5] V. P. Maslov, The Complex-WKB Method for Nonlinear Equations I: Linear Theory, Birkhäuser, Boston 1996.

[6] J. V. Ralston, Comm. Math. Phys. 51, 219 (1976).

[7] Yu. A. Kravtsov, Geometrical Optics in Engineering Physics, Alpha Science, 2005.

[8] E. Mazzucato, Phys. Fluids B 1, 1855 (1989).

[9] A. G. Peeters, Phys. Plasmas 3, 4386 (1996).

[10] G. V. Pereverzev, Reviews of Plasma Physics 19, B. B. Kadomtsev ed., Consultants Bureau, New York, 1996, p.1.

[11] G. V. Pereverzev, Phys. Plasmas 5, 3529 (1998).

[12] G. V. Pereverzev, Nucl. Fusion 32, 1091 (1992).

[13] N. Bertelli, G. V. Pereverzev, E. Poli, Proceedings of the 34th EPS Conf. on Plasma Physics, Vol 31F (ECA), P5.051 (2007).

[14] S. Nowak, A. Orefice, Phys. Fluids B 5, 1945 (1993).

[15] E. Poli, A. G. Peeters, G. V. Pereverzev, Comp. Phys. Comm. 136, 90 (2001).

[16] D. Farina, Fusion Sci. Technol. 52, 154 (2007).

[17] V. A. Fock, Electromagnetic Diffraction and Propagation Problems, Pergamon Press, New York, 1965.

[18] E. J. Heller, J. Chem. Phys. 65, 4979 (1976), and references therein.

[19] R. G. Littlejohn, Phys. Rev. Lett. 61, 2159 (1988).

[20] M. M. Popov, Ray Theory and Gaussian Beam methods for Geophysicists, Universidade Federal da Bahia, 2002.

[21] E. Poli, Fusion Sci. Technol. 53, 1 (2008). 
[22] G. D. Conway, C. Troester, J. Schirmer, C. Angioni, E. Holzhauer, F. Jenko, F. Merz, E. Poli, B. Scott, W. Suttrop, the ASDEX Upgrade Team, Proc. 8th Intl. Reflectometry Workshop IRW8, St. Petersburg, May 2007, http://plasma.ioffe.ru/irw8/proceedings.html

[23] M. A. Henderson, R. Heidinger, D. Strauss, R. Bertizzolo, A. Bruschi, R. Chavan, E. Ciattaglia, S. Cirant, A. Collazos, I. Danilov, F. Dolizy, J. Duron, D. Farina, U. Fischer, G. Gantenbein, G. Hailfinger, W. Kasparek, K. Kleefeldt, J.-D. Landis, A. Meier, A. Moro, P. Platania, E. Poli, G. Ramponi, G. Saibene, F. Sanchez, O. Sauter, A. Serikov, H. Shidara, P. Spaeh, V. S. Udintsev, H. Zohm, C. Zucca, Nucl. Fusion 48, 054013 (2008).

[24] G. Ramponi, D. Farina, M. A. Henderson, E. Poli, G. Saibene, O. Sauter, H. Zohm, C. Zucca, Nucl. Fusion 48, 054012 (2008).

[25] A. A. Balakin, M. A. Balakina, G. V. Permitin, A. I. Smirnov, J. Phys. D: Appl. Phys. 40, 4285 (2007).

[26] A. A. Balakin, M. A. Balakina, E. Westerhof, Nucl. Fusion 48, 065003 (2008).

[27] C. Honoré, P. Hennequin, A. Truc, A. Quéméneur, Nucl. Fusion 46, S809 (2006).

[28] V. L. Ginzburg, Propagation of electromagnetic waves in plasma, Translated from the Russian by Royer and Roger; edited by Walter L. Sadowski, Gordon and Breach, Inc., New York, 1961.

[29] M. Abramowitz and I. A. Stegun, Handbook of Mathematical Functions, National Bureau of Standards, Applied Mathematics Series 55.

[30] Yu. A. Kravtsov and P. Berczynski, Stud. Geophys. Geod. 51, 1-36 (2007).

[31] V. V. Bulanin and M. V. Yafanov, Plasma Physics Reports 32, 47 (2006).

[32] R. G. Littlejohn and W. G. Flynn, Phys. Rev. A 44, 5239 (1991).

[33] R. Prater, D. Farina, Yu. Gribov, R. W. Harvey, A. K. Ram, Y.-R. Lin-Liu, E. Poli, A. P. Smirnov, F. Volpe, E. Westerhof, A. Zvonkov and the ITPA Steady State Operation Topical Group, Nucl. Fusion 48, 035006 (2008).

[34] Since in this Section we consider a homogeneous medium (apart from the jump in the absorption coefficient across the boundary $x=0$ ), we will not introduce here a scale length $L$ and corresponding dimensionless coordinates as in Sec. III. The role of the dimensionless parameter $\kappa$ is played in Eq. (28) by the vacuum wavevector $k_{0}$. 